\title{
Comparison of In-Plane and Out-of-Plane Failure Modes of Masonry Arch Bridges using Discontinuum Analysis
}

\section{Bora Pulatsu', Ece Erdogmus², and Paulo B. Lourenço ${ }^{3}$}

Abstract: This research aims to provide a better understanding of the structural behavior of masonry arch bridges using advanced modeling strategies. Two main contributions are achieved in this article; first, triggering mechanisms for the out of plane failure of spandrel walls are established; second, the influence of soil backfill on the behavior and strength of the bridges is presented through a comprehensive parametric study. Here, masonry arch bridges are modeled using a discontinuum approach, composed of discrete blocks, including also a continuum mesh to replicate infill material, adopting a framework of discrete element modeling. The equations of motion for each block are solved by an explicit finite-difference method, using the commercial software 3DEC. The results of the preliminary analyses are compared with analytical solutions and limit state analysis for validation purposes. Different arch bridge models, representing common geometrical properties in the northwest lberian Peninsula are analyzed. Transverse effects, damage patterns and collapse mechanisms are discussed under different types of loading. The analysis demonstrated the severe capacity reduction due to spandrel wall failures and the importance of soil backfill in results, only possible by taking advantage of the performed numerical modeling strategy.

\footnotetext{
${ }^{1} \mathrm{PhD}$ Candidate, Durham School of Architectural Engineering and Construction, University of NebraskaLincoln, Nebraska, United States, bpulatsu@unomaha.edu

2 Professor, Durham School of Architectural Engineering and Construction, University of NebraskaLincoln, Nebraska, United States, eerdogmus2@unl.edu

3 Professor, ISISE, Department of Civil Engineering. University of Minho, Guimaraes, Portugal, pbl@civil.uminho.pt
} 


\section{Introduction}

Masonry arch bridges constitute an important asset of the transportation infrastructure not only in Europe but also in northeastern United States [1]. Although, newer construction materials, such as steel or reinforced concrete, became more popular in the construction sector after the mid-20 ${ }^{\text {th }}$ century, stone and brick masonry bridges still continue to make a remarkable contribution to world's infrastructure. For instance, masonry arch bridges constitute around $40 \%$ of the bridge stock in Europe [2]. In United States, there are 1700 masonry bridges, according to the 2013 National Bridge Inventory (NBI) [3]. Given that the majority of these bridges are older than 100 years [4], they may suffer from material deterioration, lack of maintenance, increased axle loads and high volume of traffic through the decades.

Notwithstanding, a remarkable portion of masonry bridges still constitute the heritage of transportation system in many countries. Therefore, preserving their structural integrity has a critical importance due to their historical significance. On the other hand, it has been a challenge for engineers to analyze masonry arch bridges because there are no widely adopted structural analysis procedures, and true understanding of their complex behavior requires nonlinear analysis. Furthermore, there are numerous parameters affecting the strength, stiffness and overall collapse mechanism of masonry bridges such as: boundary conditions, backfill properties, span, rise and arch thickness among others.

In this research, a vulnerable local failure mechanism, namely spandrel wall collapse, and global failure mode of arch barrel are investigated. In addition, a parametric study is performed on the backfill properties and the obtained results are compared with a simplified approach. The capacity and the transverse failure of spandrel walls are simulated by means of three-dimensional mixed discrete-continuum approach. 


\section{Background on Numerical Analysis of Masonry Arch Structures}

It is in the last few decades that advances in computer science and engineering made it possible to implement numerical methods to solve the governing differential equations. Briefly, numerical modelling of masonry structures may be examined under two main categories: continuum and discontinuum approaches.

\subsection{Continuum based models}

In continuum models, the properties of masonry components (units and mortar) are averaged and an equivalent homogeneous medium is generated. Therefore, an averaged constitutive relationship is required to use in the numerical model. The continuum parameters may be determined from experimental tests, but specimens should have a reasonably large scale to yield homogeneous state of stress, or homogenization techniques [5]. The finite element method (FEM) is one of the most common numerical modeling strategies employed in continuum-based models, i.e. macro modeling approach, with different levels of complexity.

The least complicated way to model a masonry arch may be achieved via onedimensional beam-type elements implemented into the framework of traditional finite element procedure. Boothby [6] used frame elements to perform load rating for different bridges and validated his linear elastic solution via field tests. Furthermore, the elastic perfectly plastic in compression and no tensile resistant (NTR) material model for masonry was used by Brencich and De Francesco [7] and an iterative procedure was implemented to adjust the effective height of the arch without allowing tensile stresses. They applied the same procedure on multi span masonry arch bridges and validated the results via experimental studies from the literature [8]. Moreover, dynamic behavior of masonry arches and arch bridges were investigated using a fiber beam approach in which effective material properties are taken into account [9]. Although, one dimensional models simplify the problem and neglect the complex fill-structure interaction, they have a strong advantage in terms of computational cost compared with two and three-dimensional models. 
Backfill material may be represented in two dimensional models by explicitly incorporating an elastoplastic constitutive law for the soil [10]. Additionally, to introduce discontinuities at the joints, unilateral contact interfaces may be used in the arch, which allow to obtain joint openings, cracks and sliding failure [11]. However, 2D simulations do not provide information about transverse direction since the numerical model is generally taken as a symmetric cross section with respect to span direction. Hence, they may not represent inherent three-dimensional response of masonry arch structures [12]. On the other hand, three-dimensional models capture the full structural response and local failures, such as spandrel wall collapse and skew arch bridge behavior. Fanning and Boothby [13] introduced a three-dimensional nonlinear finite element analysis to predict the stone arch bridge behavior by comparing the results of full-scale field test on existing bridges. They emphasized the importance of three-dimensional effects and highlighted the relative deflection (sliding or movement) between backfill and masonry system (arch barrel and spandrel wall). Furthermore, Conde et al. [14] noted critical transverse effects while assessing the load carrying capacity of Vilanova Bridge. Hence, the importance of soil backfill on the transverse effects of masonry arch bridges have been stated by researchers and noticeable effort has been spent to simulate this complex behavior via numerical solutions.

\subsection{Discontinuum Modeling}

The traditional continuum-based calculation techniques, usually FEM based, are not able to reflect the complex mechanical interaction of materials or structures consisting of discontinuous system, i.e. dry joint masonry structures, unless interface elements are not considered $[15,16]$. The discrete element method (DEM) provides a naturally discontinuous medium where complex mechanical interaction of distinct blocks can be simulated. Masonry construction, consisting of stiff units (stone or brick) and weak-or no mortar joints, fits the formulation of DEM which provides quite realistic simulations in terms of failure mechanism. Thus, discontinuum idealization provides significant advantages to model complex structures, which may experience complete block separation and large movements [17]. Depending on the complexity of the problem and accuracy needed, large number of discrete blocks can be used to represent each 
This is a pre-print version of an article published in Engineering Structures. Pulatsu, B., Erdogmus, E., \& Lourenço, P. B. (2019). Comparison of in-plane and out-of-plane failure modes of masonry arch bridges using discontinuum analysis. Engineering Structures, 178, 24-36.

masonry unit individually, or a single discrete block may represent more than one unit. Hence, the number of the blocks in the numerical model should be adjusted between computational cost and realistic structural behavior.

It is worth noting that in the last few decades, remarkable developments have been achieved in the modeling of masonry arch structures using DEM and similar discontinuous modeling strategies. As an example, Lemos [18] presented the load carrying capacity of 2D and 3D masonry arch bridge models by means of discrete element method without fill-structure interaction. The results indicated the contributions of the spandrel walls on the load bearing capacity of masonry arch bridges. Continuum/discontinuum based computational techniques were investigated to analyze backfilled masonry arch bridges by Thavalingam et. al. [19] using three different modeling strategies. The capabilities and limitations of each examined model were indicated through sensitivity analysis. Additionally, the relative importance of the material and interface properties (backfill-arch interaction) were discussed. Discontinuous deformation analysis (DDA) of the Bridgemill arch was presented by Bićanić et al. [20] in which deformable blocks were used to model soil backfill that were arbitrarily discretized and interacted through joint interfaces via Mohr Coulomb constitutive law with tension cut-off. There were no cohesion and tensile strength assigned at the voussoir interfaces in the model and the influence of lateral constraint on the carrying capacity of arch bridge was discussed. Tóth et al. [21] carried out a comprehensive parametric analysis on the backfill properties discussing the effect of each parameter on the strength and deformability of a single and multi-span stone masonry arch bridges. The behavior and capacity of false skew masonry arch structures composed of various geometrical proportions (shallow, semi-shallow and deep) were presented by Sarhosis et al. [22] and the detrimental influence of different skew angles was presented on each false skew arch model. Recently, authors presented a simplified discrete element modeling strategy [23] and performed experimentally validated study on a scaled masonry arch bridge model [24]. Dead load of the backfill material was considered explicitly, however, the backfill/structure interaction were neglected, while arch barrel and spandrel walls were modeled via rigid blocks along with the appropriate boundary conditions. A good agreement was achieved 
between the numerical model and the experimental study, in terms of collapse mechanism and load carrying capacity.

\subsection{Theoretical Background of DEM}

According to Cundall and Hart [25], one discrete element code should provide several features, namely: I) the possibility to simulate large displacement, rotation and sliding failure of individual blocks; ii) automatic contact detection. Further information on contact detection algorithm for polyhedral blocks can be found in [26].

In present research, each masonry unit is considered as a rigid block with six degrees of freedom ( 3 rotational and 3 translational). The discontinuous system of blocks has mechanical interaction acting through their sub-contacts with each other where the interface forces develop and are updated according to the assigned failure criteria. The elastic normal and shear force increments are calculated as given in Equation 1 and 2, respectively.

$$
\begin{gathered}
\Delta F_{n}=k_{N} \Delta U_{N} A_{C} \\
\Delta F_{S}=k_{S} \Delta U_{S} A_{C}
\end{gathered}
$$

where, $k_{N}, k_{S}$ and $A_{C}$ are the stiffness in the normal direction, stiffness in shear direction and the area of sub-contact. Furthermore, $\Delta F_{n}, \Delta F_{S}, \Delta U_{N}$ and $\Delta U_{S}$ indicate the resultant normal force, resultant shear force, normal and shear displacement increments, respectively. Once the force increments are calculated, the total normal and shear force vectors are updated.

The obtained contact forces are adjusted according to the assigned contact constitutive model. In this research, the contact normal force is limited by the tensile strength $f_{T}$ as given in Equation 3. Moreover, in shear the direction, Coulomb-slip joint model is utilized, and shear forces are limited according to Equation 4.

$$
F_{n} \leq f_{T} A_{C}
$$




$$
F_{s} \leq c A_{C}+F_{n} \tan \phi
$$

where, $c$ and $\phi$ are the joint cohesion and friction angle, respectively. A brittle contact model is assumed at the joints by setting the tensile strength and cohesion to zero onset of failure.

The governing translational and rotational differential equations for rigid blocks are solved explicitly at each time-step and the constitutive equations are applied. First, the equations of translational motion for a single block is given in Equation 5 .

$$
\ddot{x}_{i}+\alpha \dot{x}_{i}=\frac{\Sigma F_{i}}{m_{i}}+g_{i}
$$

where, $\ddot{x}, \dot{x}, \alpha, m$ and $g_{i}$ are the acceleration of the block centroid, the velocity of the block centroid, the viscous damping constant, the block mass and the gravity acceleration vector, respectively. The sum of forces acting on a block is indicated as $\Sigma F$ which includes contact and external forces. In addition, the simplified rotational equation of motion is presented in Equation 6.

$$
\dot{\omega}_{i}+\alpha \omega_{i}=\frac{\Sigma M_{i}}{I}
$$

where $\omega, I$ and $\Sigma M$ represent the angular velocity, moment of inertia and total torque. The given equations of motion are integrated by a central difference procedure, in which translational and rotational velocities are calculated. The time step, $t$, is divided into mid intervals and next time step $t+\frac{\Delta t}{2}$ is represented as $t^{+}$and the previous time step $t-\frac{\Delta t}{2}$ is indicated as $t^{-}$. Once, the velocities are obtained for the centroid of a rigid block, translational and rotational increments are calculated. Accordingly, the positions of the blocks are updated. Then, contact-displacement increments are employed in the next time step to calculate the new contact forces using the force-displacement law.

A similar numerical procedure is performed for the backfill material, however, in this case, a continuum deformable block is considered, which is internally discretized into tetrahedral elements interacting with its surrounding, i.e. arch barrel and spandrel walls, at the boundary surfaces through contact points. Note that vertices of the tetrahedral 
This is a pre-print version of an article published in Engineering Structures. Pulatsu, B., Erdogmus, E., \& Lourenço, P. B. (2019). Comparison of in-plane and out-of-plane failure modes of masonry arch bridges using discontinuum analysis. Engineering Structures, 178, 24-36.

elements are referred as gridpoints, whereas tetrahedral elements are designated as zones. The deformation of the continuous deformable block is described by the nodal displacements. Hence, velocities at the gridpoints may be calculated as

$$
\begin{gathered}
\dot{u}_{i}\left(t^{+}\right)=\dot{u}_{i}\left(t^{-}\right)+\frac{\Delta t}{m_{n}}\left\{\Sigma F_{i}^{t}-\left(F_{d}\right)_{i}\right\} \\
\Sigma F_{i}=F_{\text {gravity }}+F_{\text {contact }}+F_{\text {external }}+F_{\text {internal }} \\
\left(F_{d}\right)_{i}=\alpha\left|\Sigma F_{i}^{t}\right| \operatorname{sgn}\left(\dot{u}_{i}\left(t^{-}\right)\right)
\end{gathered}
$$

where $m_{n}, \Sigma F_{i}$ and $F_{d}$ are the nodal mass, the total nodal force vector calculated at each gridpoint and the damping force. The total force vector includes gravity forces $F_{\text {gravity }}$, sub-contact forces $F_{\text {contact }}$, external applied loads $F_{\text {external }}$, and contribution to the internal stresses in the zones adjacent to the grid point $F_{\text {internal }}$, obtained as

$$
F_{\text {internal }}=\int_{S} \sigma_{i j} n_{j} d s
$$

where, $n_{j}$ stands for the normal to the surface and $\sigma_{i j}$ indicates the zone stress tensor.

The Mohr-Coulomb yield criterion is used for the zones and stress tensors are updated in the framework of an explicit dynamic-solution scheme. For each time step, elastic trial (or guess) stresses are calculated using principal stresses $\left(\sigma_{1}, \sigma_{2}, \sigma_{3}\right)$ and strain increments $\left(\Delta \varepsilon_{1}, \Delta \varepsilon_{2}, \Delta \varepsilon_{3}\right)$ (one example is given in Equation 11, while the rest can be obtained by a rotation of indices in the stress and strain components) and checked against composite failure criterion.

$$
\sigma_{1}^{i+1}=\sigma_{1}^{i}+\alpha_{1} \Delta \varepsilon_{1}+\alpha_{2}\left(\Delta \varepsilon_{2}+\Delta \varepsilon_{3}\right)
$$


This is a pre-print version of an article published in Engineering Structures. Pulatsu, B., Erdogmus, E., \& Lourenço, P. B. (2019). Comparison of in-plane and out-of-plane failure modes of masonry arch bridges using discontinuum analysis. Engineering Structures, 178, 24-36.

where, $\alpha_{1}$ and $\alpha_{2}$ are equal to $K+4 G / 3$ and $K-2 G / 3$, in which $K$ and $G$ indicate bulk and shear modulus. The stress states that belong to the next time step is demonstrated using the superscripts, $i+1$.

Mohr-Coulomb model with a Rankine tension cut-off is used, meaning that elastic guess may violate shear or tensile yield functions. The failure envelope, given in the principle stress space, is defined for shear and tension yield functions in Equation 12 and Equation 13, respectively.

$$
\begin{gathered}
f^{s}=\sigma_{1}-\sigma_{3} N_{\phi}+2 c \sqrt{N_{\phi}} \\
f^{t}=\sigma^{t}-\sigma_{3}
\end{gathered}
$$

where principle stresses are ordered as $\sigma_{1} \leq \sigma_{2} \leq \sigma_{3}$ and $c, \phi$ are the cohesion strength and friction angle. Moreover, $\sigma^{t}$ represents tensile strength and $N_{\phi}$ is calculated as

$$
N_{\phi}=\frac{1+\sin \phi}{1-\sin \phi}
$$

The associated flow rule is used for tension but a nonassociated flow rule for the shear potential. Once the material becomes plastic, the stress state is corrected with the new stress state by mapping it back to the yield surface. Therefore, the initial elastic estimates are replaced, if any, by plastic corrections. The plastic multiplier $\lambda^{s}$ (Equation 15) can be calculated for the new stress points $\sigma_{i}^{N}$ (Equation 16), which are located on the yield shear surface and satisfies $f^{s}=0$. The elastic guess is represented as $\sigma_{i}^{I}$.

$$
\begin{gathered}
\lambda^{s}=\frac{f^{s}\left(\sigma_{1}^{I}, \sigma_{3}^{I}\right)}{\left(\alpha_{1}-\alpha_{2} N_{\psi}\right)-\left(\alpha_{2}-\alpha_{1} N_{\psi}\right) N_{\phi}} \\
\sigma_{1}^{N}=\sigma_{1}^{I}-\lambda^{s}\left(\alpha_{1}-\alpha_{2} N_{\psi}\right) \\
\sigma_{2}^{N}=\sigma_{2}^{I}-\lambda^{s} \alpha_{2}\left(1-N_{\psi}\right) \\
\sigma_{3}^{N}=\sigma_{3}^{I}-\lambda^{s}\left(-\alpha_{1} N_{\psi}+\alpha_{2}\right)
\end{gathered}
$$


and $N_{\psi}$ is calculated as

$$
N_{\psi}=\frac{1+\sin \psi}{1-\sin \psi}
$$

The plastic multiplier $\lambda^{t}$ may be computed as, simply, $f^{t}\left(\sigma_{3}^{I}\right) / \alpha_{1}$, and new stresses (or corrected) are found as

$$
\begin{aligned}
& \sigma_{1}^{N}=\sigma_{1}^{I}+\lambda^{t} \alpha_{2} \\
& \sigma_{2}^{N}=\sigma_{2}^{I}+\lambda^{t} \alpha_{2} \\
& \sigma_{3}^{N}=\sigma_{3}^{I}+\lambda^{t} \alpha_{1}
\end{aligned}
$$

Thus, the dynamic procedure explained above is followed by using a mixed approach where rigid and deformable blocks are employed in the same model. To reach the static solutions, dynamic effects are damped in order to converge to an equilibrium state using a local form of adaptive damping (further information can be found in [27]). It is important to note that the implemented numerical strategy is conditionally stable, if the time step $\Delta t$ is kept below a critical time step, which may be estimated as

$$
\Delta t_{n}=(\text { fract }) 2 \max \left(\sqrt{\frac{m}{k}}\right)
$$

where fract, $m$ and $k$ are user-supplied value (taken as 0.1 by default), mass of the smallest block in the system and the maximum contact stiffness. Moreover, scaled masses are used to improve convergence. Note that as the contact stiffness increases, the time step decreases, so very rigid contacts provide extensive computation times.

\section{Discrete Element Validation for Masonry Arches}

\subsection{Number of Blocks and Contact Stiffness}

The validation of discrete element model plays a crucial role to obtain realistic results and simulations. In DEM, there are two main questions that need to be addressed. 
This is a pre-print version of an article published in Engineering Structures. Pulatsu, B., Erdogmus, E., \& Lourenço, P. B. (2019). Comparison of in-plane and out-of-plane failure modes of masonry arch bridges using discontinuum analysis. Engineering Structures, 178, 24-36.

- What is the appropriate number of blocks to represent the behavior of the structure?

- What are the mechanical properties for the contacts that represent their forcedisplacement behavior?

Both questions should also take into account the computational cost and the expected accuracy from the numerical model. First, in order to demonstrate the influence of number of blocks and contact stiffness, a parametric study is performed, as previously done by researchers on different masonry strucutres [28,29]. In this study, two masonry arch forms, shallow and deep, are taken into consideration to find their lower bound arch thicknesses under their self-weight. The rise to span ratios, $r / s$, of the examined arches are 0.23 and 0.5 for shallow and deep arches, respectively. A radius of 2 meters arch (passing through the center-line of the arch) is used in both models. In Figure 1, discrete element arch models composed of 10 blocks are given.

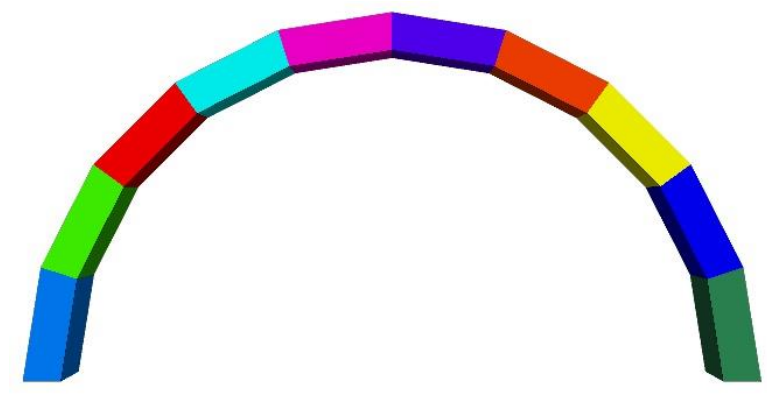

a) Circular arch composed of 10 blocks

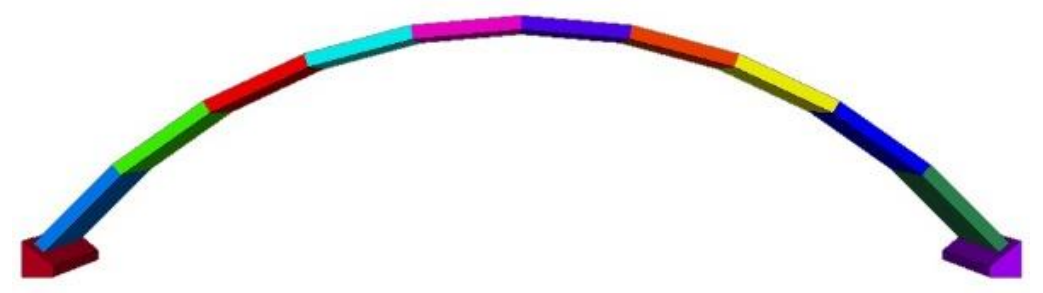

b) Shallow arch composed of 10 blocks

Figure 1. Masonry arches, $r / s: 0.5$ and $r / s: 0.23$, discrete element models. 
The numerical approximations are then compared with analytical solutions available in the literature sharing the same geometrical properties. The comprehensive research published by Cocchetti et al. [30] provides the derivation of different solutions on the minimum arch thickness with different embrace angles. In this study, the analytical solution, CCR, is compared with the numerical approximations comprising of various number of blocks and contact stiffness. Briefly, the CCR solution is obtained from the static equilibrium approach by reviewing the tangent condition of the thrust line decision of Heyman with the true line of thrust (locus of pressure points). For the derivation of the equations and detailed explanation, readers are referred to [30].

Here, the minimum arch thicknesses are found using discrete element models by gradually decreasing the arch thickness until its collapse. Additionally, contact stiffness is varied from $1 \mathrm{E}+9$ to $1 \mathrm{E}+12 \mathrm{~Pa} / \mathrm{m}$ for different number of blocks. The obtained results for both shallow and deep arches are compared with the analytical solutions, shown in Figure 2-a and Figure 2-b, respectively. The results of the analyses indicated that low contact stiffness (i.e. $1 \mathrm{E}+9$ ) yields reasonably high thickness values, while this can be partly compensated with less number of blocks. The evident observation is that if the stiffness is low or the number of blocks is high, the elastic behavior of the structure becomes relevant, deviating from the rigid-limit analysis in which the usual theory is based. A good approximation is obtained using high contact stiffness (i.e. $1 \mathrm{E}+11,1 \mathrm{E}+12$ ) using 40,70 and 100 blocks for both arch geometries. In this case, a low number of blocks defines the location of the possible hinges, presenting therefore a slightly higher capacity than the theory or, equivalently, a lower minimum arch thickness. According to the present study, it is decided to use 40 blocks with $1 \mathrm{E}+10 \mathrm{~Pa} / \mathrm{m}$ in the rest of numerical models, which provides acceptable accuracy with reasonable computational cost. 
This is a pre-print version of an article published in Engineering Structures. Pulatsu, B., Erdogmus, E., \& Lourenço, P. B. (2019). Comparison of in-plane and out-of-plane failure modes of masonry arch bridges using discontinuum analysis. Engineering Structures, 178, 24-36.

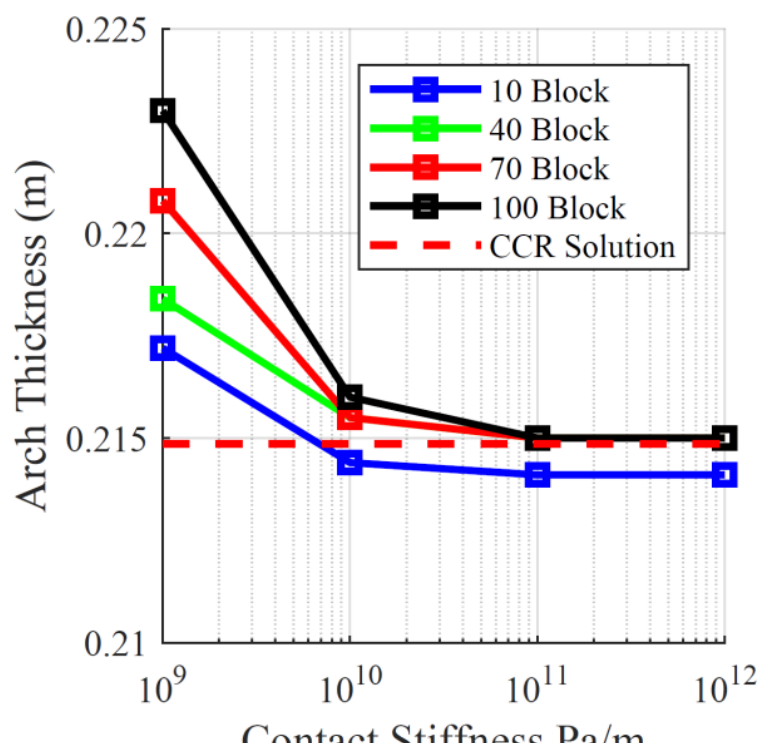

Contact Stiffness $\mathrm{Pa} / \mathrm{m}$

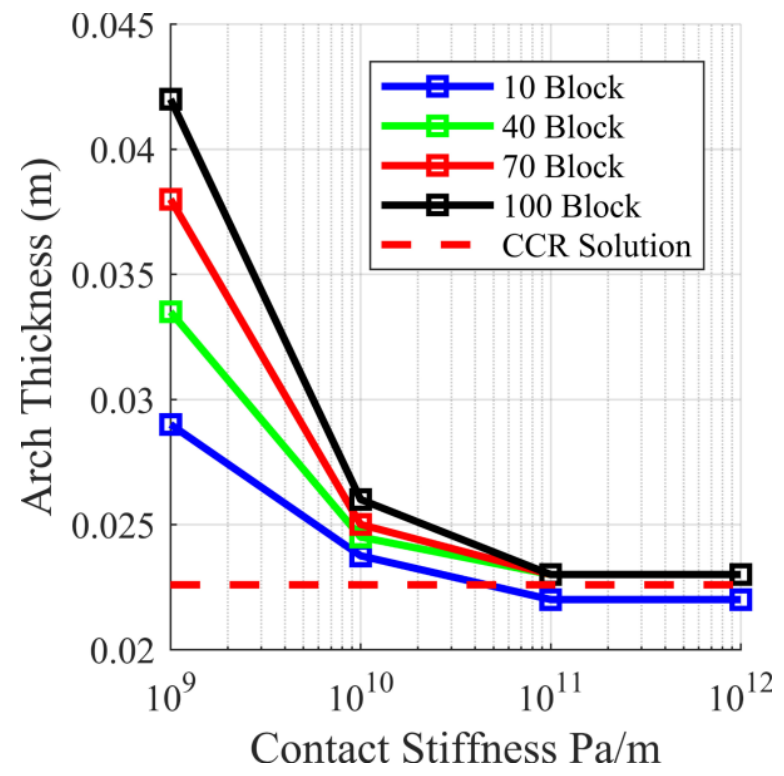

b) Minimum arch thicknesses for shallow arch with different number of blocks and contact stiffnesses. a) Minimum arch thicknesses for deep arch with different number of blocks and contact stiffnesses.

Figure 2. Parametric study on number of blocks and contact stiffness compared with analytical solutions.

It is important to note that the minimum thickness of the shallow arch is derived theoretically without any concern on practical applications. Additionally, the high contact stiffnesses is used only to verify the convergence of results against the theoretical solutions. In practice, the contact parameters should also correspond to the overall deformability of the structure. In that context, the influence of the relationship between the number of blocks and contact stiffnesses on the approximate equivalent Young's Modulus of the structure is shown in Table 3-1. The approximate equivalent elastic stiffness $\left(E_{e q}\right)$ of the discrete element model may be calculated as

$$
E_{\text {eq }}=k_{N} \cdot l_{\text {Block }}
$$

where $l_{\text {Block }}$ represent the length of rigid block (voussoir). It is worth noting that high number of blocks increase the deformability of the structure which effects the stiffness of the model. 
This is a pre-print version of an article published in Engineering Structures. Pulatsu, B., Erdogmus, E., \& Lourenço, P. B. (2019). Comparison of in-plane and out-of-plane failure modes of masonry arch bridges using discontinuum analysis. Engineering Structures, 178, 24-36.

Table 3-1. Approximate equivalent Young' Modulus, $E_{e q}(\mathrm{~Pa})$

\begin{tabular}{|c|c|c|c|c|}
\hline $\boldsymbol{k}_{\boldsymbol{N}} \backslash$ Number of Blocks & $\mathbf{1 0}$ & $\mathbf{4 0}$ & $\mathbf{7 0}$ & $\mathbf{1 0 0}$ \\
\hline $1 \mathrm{E}+9$ & $6.26 \mathrm{E}+8$ & $1.57 \mathrm{E}+8$ & $8.98 \mathrm{E}+7$ & $6.28 \mathrm{E}+7$ \\
\hline $1 \mathrm{E}+10$ & $6.26 \mathrm{E}+9$ & $1.57 \mathrm{E}+9$ & $8.98 \mathrm{E}+8$ & $6.28 \mathrm{E}+8$ \\
\hline $1 \mathrm{E}+11$ & $6.26 \mathrm{E}+10$ & $1.57 \mathrm{E}+10$ & $8.98 \mathrm{E}+9$ & $6.28 \mathrm{E}+9$ \\
\hline
\end{tabular}

\subsection{Representative Masonry Arch Bridge Modeled}

The masonry arch bridge database published by Oliveira et al. [31] is taken as a reference for the geometrical properties of the masonry arch bridge models. The suggested reference bridge geometries, which are the representative of 59 roadway masonry arch bridges existing in the northwest Iberian Peninsula, are utilized to prepare advanced and simplified numerical models. The geometrical properties and numerical models of the reference masonry arch bridges, namely semi-shallow short span (SSS), semi-shallow medium span (SMS), deep arch short span (DSS) and deep arch medium span (DMS) are given in Table 3-2 and Figure 3, respectively.

Table 3-2. Geometrical properties of the representative masonry arch bridges (dimensions are in meters).

\begin{tabular}{c|rccc} 
& & Rise & Thickness & Span \\
\cline { 5 - 5 } $\begin{array}{c}\text { Short Span arch } \\
\text { bridges }\end{array}$ & Semi-shallow & & & \\
& (SSS) & 1.5 & 0.5 & 5 \\
\hline \multirow{2}{*}{$\begin{array}{c}\text { Medium Span } \\
\text { arch bridges }\end{array}$} & Semi-shallow & & & \\
& Deep arch (DSS) & 2.5 & 0.6 & 5 \\
& Deep arch (DMS) & 3.0 & 0.7 & 10 \\
& 5.0 & 1.0 & 10
\end{tabular}

Granite is the main construction material (79\% of the surveyed bridges) used in the construction of these bridges among other materials, i.e. limestone, sandstone and schist. 
The infill depth above the crown is taken as 0.4 meters. The spandrel wall thickness is assumed as 0.5 for all reference models, which may vary between $0.4-1.2$ meters according to [32], and wall morphology is drawn randomly with an average masonry unit length of 0.5 meters for medium span arches and 0.35 meters for short span, see [33] for some typical stone dimensions in Portugal. All bridges are categorized as Class II, meaning light and little intense traffic (agricultural pathways and passages and certain municipal and forest roads) according to the Road Bridge Classification (RSA) in Portugal.

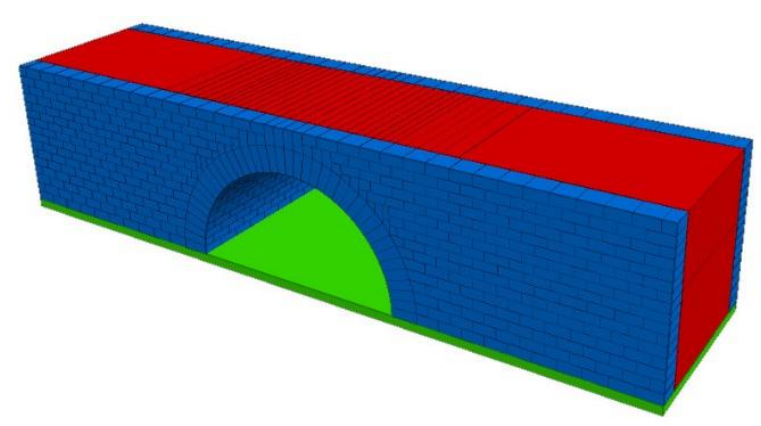

a) Deep Arch Short Span Bridge Model (DSS)

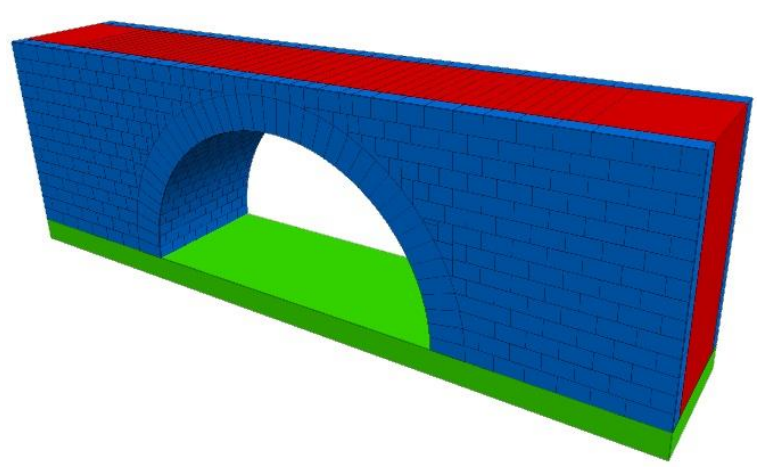

C) Deep Arch Medium Span Bridge Model (DMS)

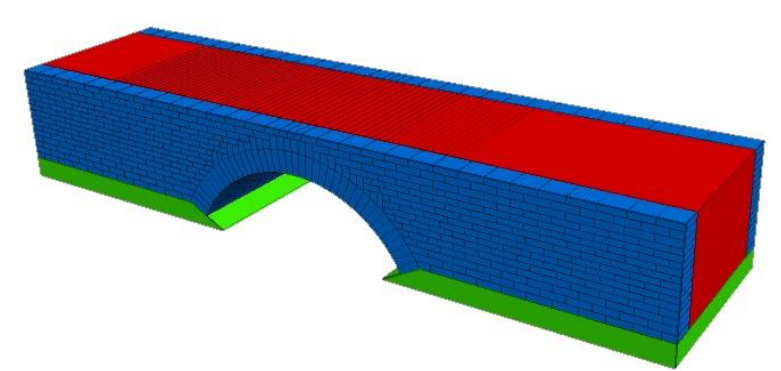

b) Semi-shallow Arch Short Span Bridge Model (SSS)

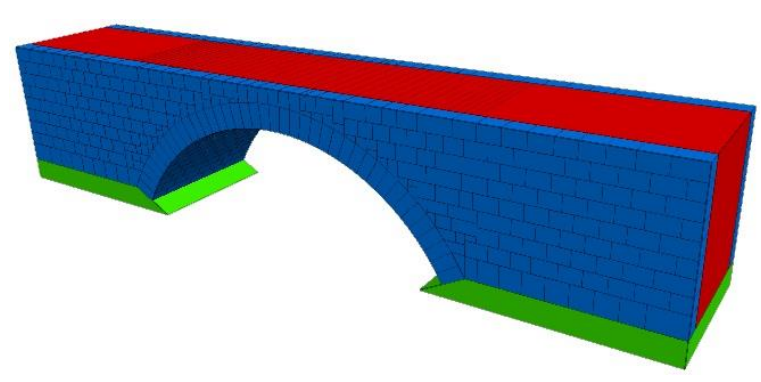

d) Semi-shallow Arch Medium Span Bridge Model (SMS)

Figure 3. Representative masonry arch bridge models. 
This is a pre-print version of an article published in Engineering Structures. Pulatsu, B., Erdogmus, E., \& Lourenço, P. B. (2019). Comparison of in-plane and out-of-plane failure modes of masonry arch bridges using discontinuum analysis. Engineering Structures, 178, 24-36.

\subsection{Comparison with Limit Analysis}

In this part, only free-standing arch barrels are analyzed using two different numerical techniques. First, computational limit analysis, initially proposed by Livesley [34], is applied. A commonly used limit state analysis (LSA) software RING, [35], is utilized to find capacity and corresponding collapse mechanisms of masonry arches. Then, the same arch models are simulated via discrete element method, using the threedimensional discrete element code, 3DEC developed by ITASCA.

It is worth to note that LSA fills the gap between empirical rules and advanced numerical solutions by providing a rapid analysis option. Furthermore, it requires much lower need of input parameters when compared with any other sophisticated solutions, i.e. nonlinear incremental analysis. Similar to DEM, masonry blocks are modeled explicitly in limit analysis considering rigid perfectly plastic material model. Thus, in both numerical approaches, masonry units are taken as rigid blocks and arch is loaded at the quarter span (across the full width) without using backfill. There is no tensile and cohesion strength are assigned at the joints. The friction angle between blocks is taken as 40 degrees to prevent any sliding failure. The obtained ultimate loads and corresponding hinging mechanisms are given in Table 3-3 and Figure 4, respectively for the arches introduced above. As expected, the two modeling techniques provided quantitative results within $2 \%$ of each other and they displayed identical kinematic mechanisms.

Table 3-3. Ultimate load carrying capacity of semi-shallow and deep arches

\section{DEC Limit Analysis Difference (\%)}

$\begin{array}{llll}\text { SMS } & 592 \mathrm{kN} & 603 \mathrm{kN} & <2 \\ \text { DMS } & 563 \mathrm{kN} & 568 \mathrm{kN} & <1\end{array}$




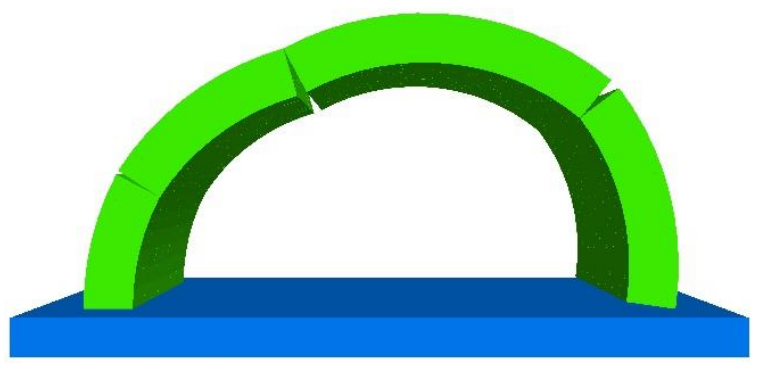

a) Failure mechanism of Deep Arch (DEM)

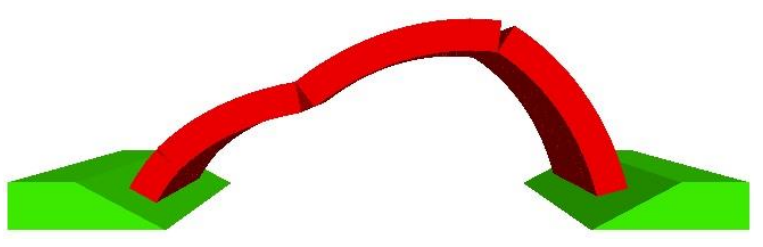

c) Failure mechanism of SMS (DEM)

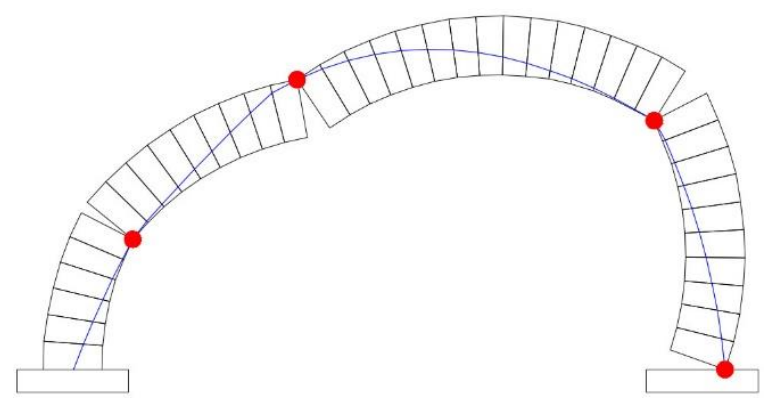

b) Failure mechanism of Deep Arch (LSA)

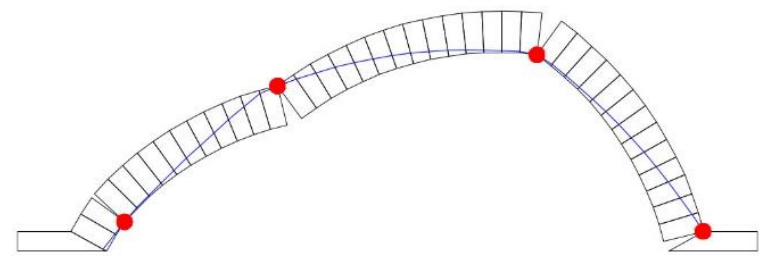

d) Failure mechanism of SMS (LSA)

Figure 4. Failure mechanism of discrete element masonry arch models loaded at the quarter span.

\section{Simulation of Masonry Arch Bridge Models}

Masonry arch bridges have different kind of fill materials (designated as backfill and usually soil), which play an important role on the strength of the bridges both by applying passive pressure and providing live load dispersion on the arch barrel [36]. Therefore, an investigation about the fill properties is necessary and beneficial to assess the capacity of masonry arch bridges. In this part, 2D simplified approach and detailed numerical models are compared. Then, the soil properties, friction angle and cohesion, are varied in both methods and compared. The influence of pavement on the road is out of the scope of this paper. Furthermore, two different live load patterns (Figure 5) are applied on the bridges and corresponding collapse mechanisms are discussed. First, a vehicle load is considered for a standard Portuguese vehicle composed of three axles spaced equally 1.5 meters apart, with a load of $100 \mathrm{kN}$ per axle and a width of 2 meters [37]. Additionally, uniform line load (i.e. knife edge) is applied along the total width between spandrel walls 
This is a pre-print version of an article published in Engineering Structures. Pulatsu, B., Erdogmus, E., \& Lourenço, P. B. (2019). Comparison of in-plane and out-of-plane failure modes of masonry arch bridges using discontinuum analysis. Engineering Structures, 178, 24-36.

as a second loading scenario. Both live load types (axles and knife edge), are applied at the critical position on the backfill which is found using a simplified in-plane analysis.

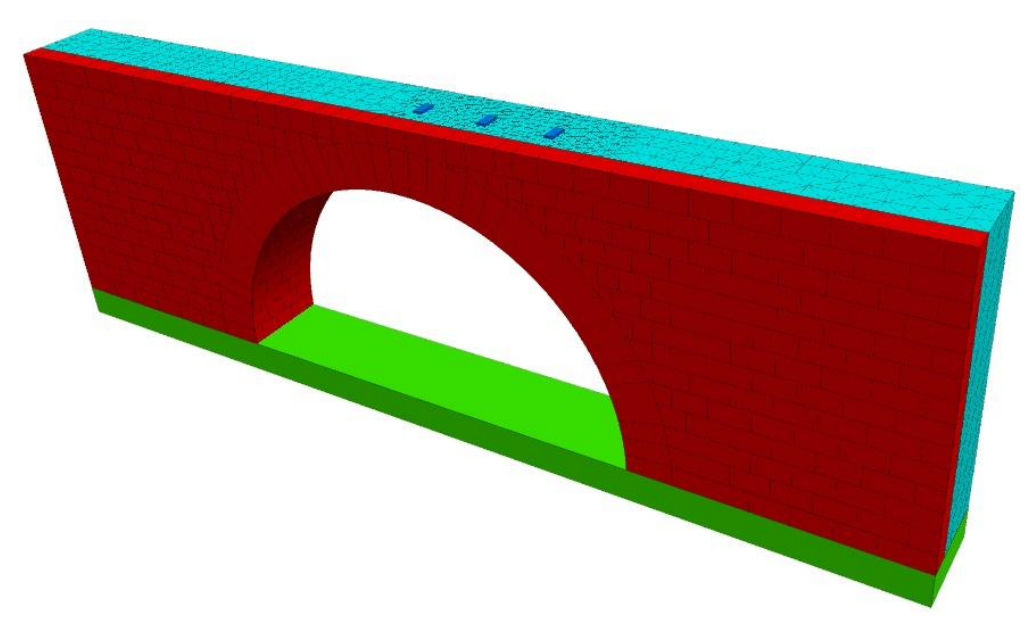

a) Axle loads applied on rigid plates (half model with a longitudinal symmetry axis)

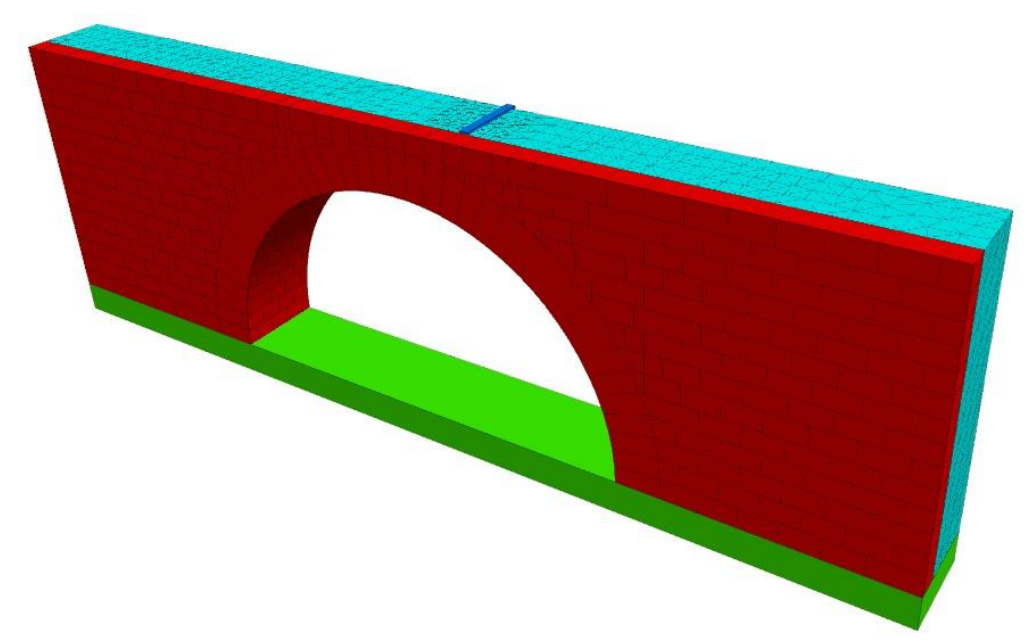

a) Knife-edge load applied through a rigid thin plate (half model with a longitudinal symmetry axis)

Figure 5. DMS under different loading patterns.

In limit state analysis, a Boussinesq type dispersion of live load with an angle of 30 degrees is considered. Moreover, passive earth pressure is modeled using one 
dimensional bar elements, or 'backfill elements', at the section of the arch barrel which sways into the fill material [35]. The classical lateral earth pressure, developed for retaining walls, is utilized based on Rankine theory. The relevant horizontal and vertical soil pressures, acting on the extrados of a voussoir, are calculated explicitly. Active and passive forces are automatically found by the RING software. On the other hand, in the detailed 3D model, a mixed discrete-continuum approach is utilized in which the fill material is modeled as a continuous finite element mesh by adopting the Mohr-Coulomb failure criterion with ideal plastic behavior. The same modeling strategy was applied to simulate the behavior and capacity of small-scale dry-joint retaining wall by Quezada et al. [38]. Here, this strategy is further extended to masonry arch bridges. Throughout this research, load-controlled analysis is performed. Local and global failure modes are observed by monitoring the in-plane deflection of the arch voussoir and out of plane deflection of the spandrel wall where the highest displacements are expected. Symmetry boundary conditions are employed through the centerline of the bridge and only the half of the bridge is modeled for computational efficiency. The translational degrees of freedom of the nodes located at both ends of the backfill material are restricted through the longitudinal direction and left free in vertical and transverse directions.

\subsection{D and 3D Modeling of Masonry Arch Bridges}

First, the semi-shallow arch bridge with short span is subjected to a line load, applied at the quarter span, using detailed and rigid block limit state models. Again, in both computational techniques, masonry units are assumed as rigid blocks, thus, all deformations are lumped at the joints. It is worth noting that severe deterioration of materials, excessive live loads and fatigue problem may yield cracks and failure in the masonry units that may affect the overall capacity of the structure. However, this research focuses on the structural defects of the soil backfilled masonry arch bridges suffering from weak mortar joints. Hence, there is no failure considered at the masonry units. The reference masonry and soil backfill parameters along with the contact properties are given in Table 4-1. It is worth noting that in limit state analysis the dead load of the spandrel walls is included with the backfill and an averaged self-weight value of $21.25 \mathrm{kN} / \mathrm{m}^{3}$ is 
This is a pre-print version of an article published in Engineering Structures. Pulatsu, B., Erdogmus, E., \& Lourenço, P. B. (2019). Comparison of in-plane and out-of-plane failure modes of masonry arch bridges using discontinuum analysis. Engineering Structures, 178, 24-36.

used. The tensile strength and cohesion values are considered as zero with a friction angle of 30 degrees at the joints between masonry units. This represents lower bound for friction. The mechanical properties of the joints at the interfaces between masonry and soil are also presented in Table 4-1.

Table 4-1. Reference material and contact properties (M-M: contact parameters between masonry and masonry, M-S: contact parameters between masonry and soil)

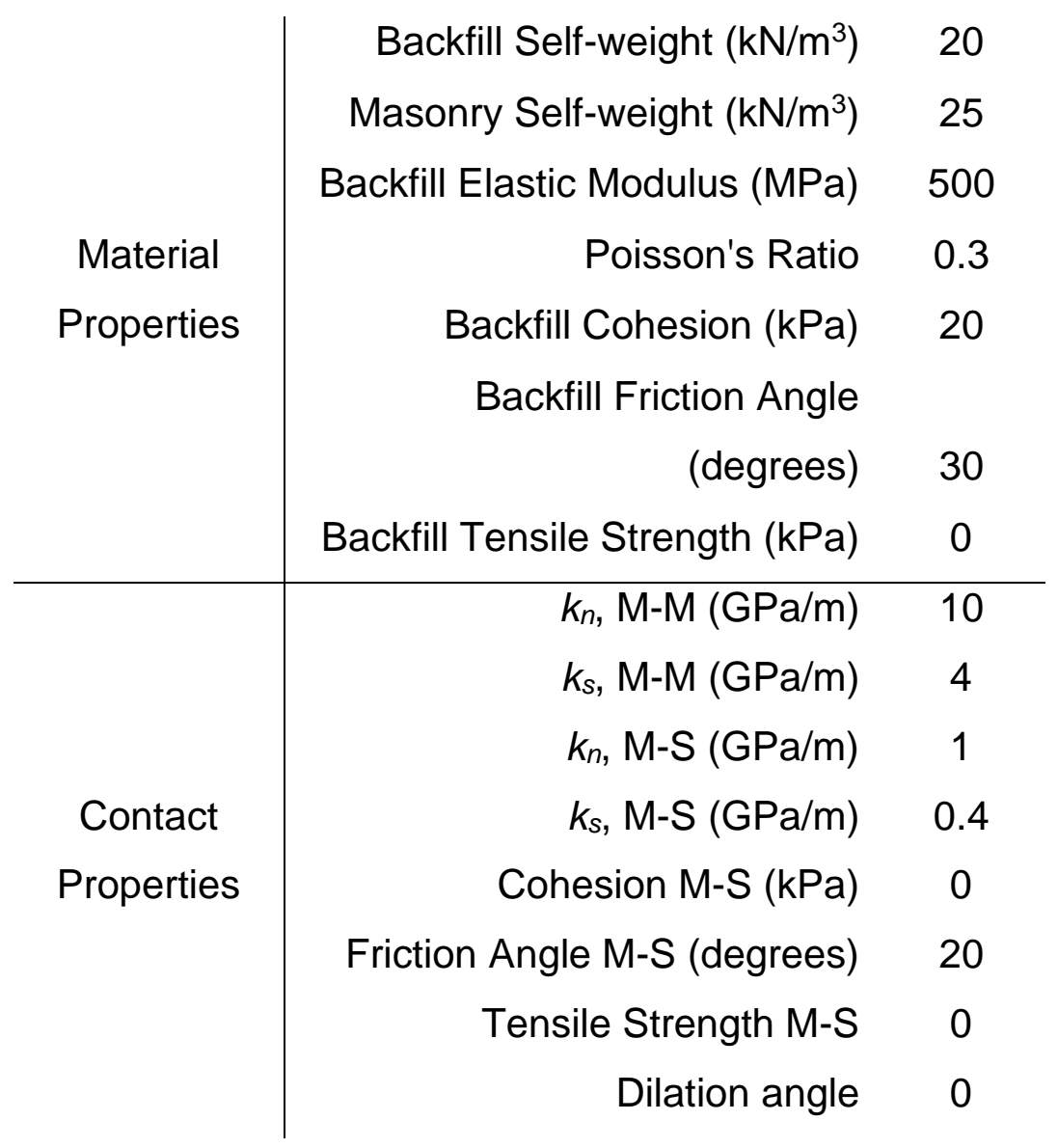

The results indicated that the detailed discontinuum analysis provided similar results when compared with the simplified two-dimensional approach. Identical hinging mechanisms are observed at the arch barrel, as shown in Figure 6. It is important to note that although out of plane movements at the spandrel wall are recorded in the 3D model during the analysis, the bridge collapsed due to in plane kinematic mechanism of the arch. 
This is a pre-print version of an article published in Engineering Structures. Pulatsu, B., Erdogmus, E., \& Lourenço, P. B. (2019). Comparison of in-plane and out-of-plane failure modes of masonry arch bridges using discontinuum analysis. Engineering Structures, 178, 24-36.

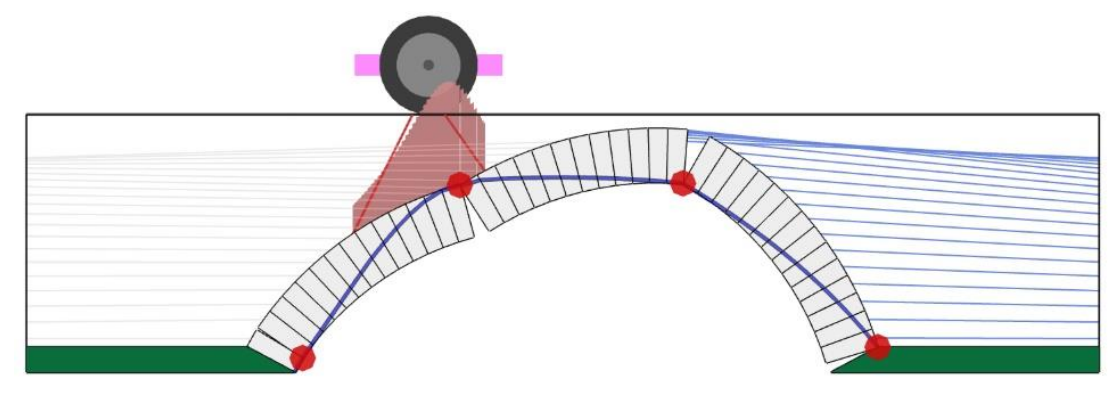

a) Collapse Mechanism (2D) Limit State Analysis (RING)

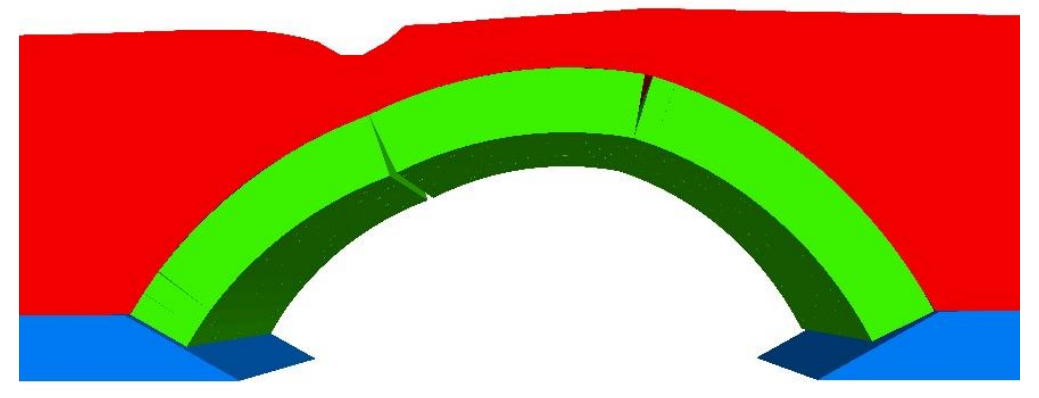

b) DEM Model (3DEC)

Figure 6. Collapse mechanism of semi shallow masonry arch bridge with short span model.

Additionally, the difference in capacity is lower than $5 \%$ as shown in Figure 7 . However, this may not be the case for all situations, since type of loading, backfill properties and geometrical characteristics of the structure may affect the collapse mechanism, hence, the load carrying capacity. 
This is a pre-print version of an article published in Engineering Structures. Pulatsu, B., Erdogmus, E., \& Lourenço, P. B. (2019). Comparison of in-plane and out-of-plane failure modes of masonry arch bridges using discontinuum analysis. Engineering Structures, 178, 24-36.

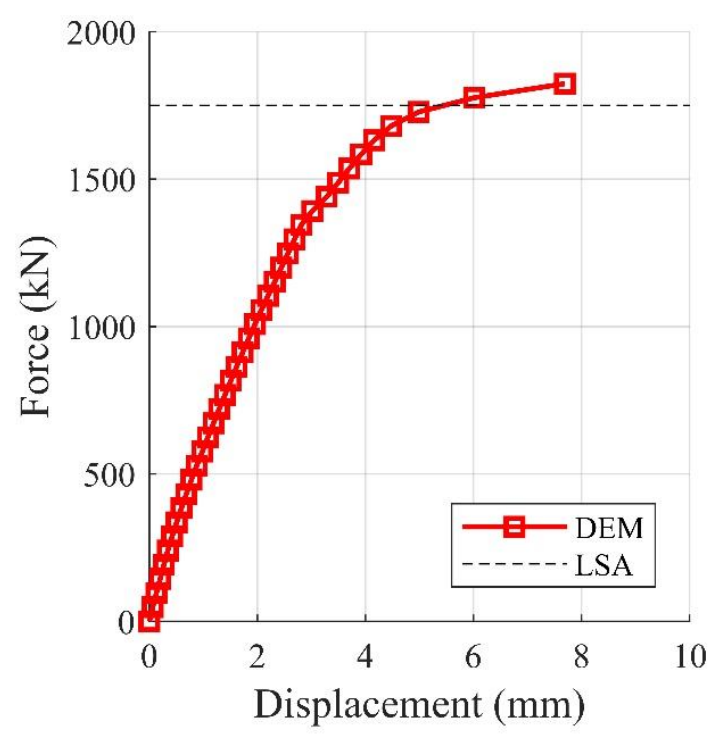

Figure 7. Comparison the results of Limit State Analysis and Discrete Element Model.

\subsection{Parametric Study on Backfill Properties}

Parametric study is performed on the mechanical properties of the backfill by varying the friction angle and cohesion in both computational methods, using the same example as before. According to the analysis, for higher cohesion values, the detailed discrete element model provided higher load carrying capacities than limit state analysis. This may be expected from the simplified approach since the backfill interaction is taken into account only approximately. On the other hand, in terms of friction angle variation, both approaches give results reasonably close to each other compared to variation in cohesion. In Figure 8, it is possible to observe the pronounced effect of the backfill on the load carrying capacity of the masonry arch bridges. The simplified approach provided conservative results, especially for the cohesive infill material. Hence, elasto-plastic formulation for the backfill material give higher load bearing capacities compared to the simplified solution, which is less sensitive to backfill properties. The obtained results are in line with the comparison between other advanced modeling strategies and simplified solutions, presented by $[39,40]$. 
This is a pre-print version of an article published in Engineering Structures. Pulatsu, B., Erdogmus, E., \& Lourenço, P. B. (2019). Comparison of in-plane and out-of-plane failure modes of masonry arch bridges using discontinuum analysis. Engineering Structures, 178, 24-36.

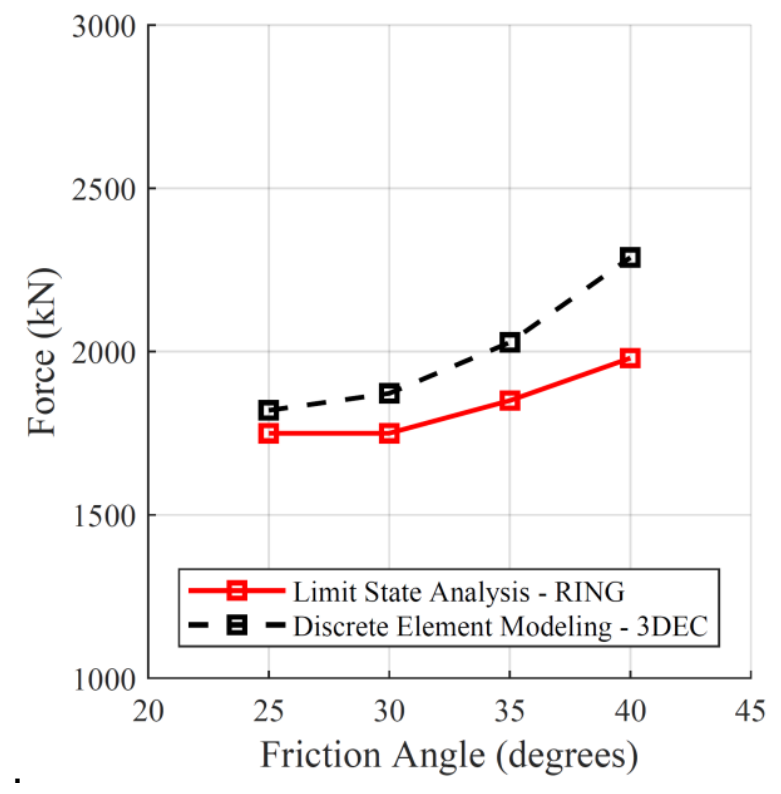

a) Influence of friction angle on the limit and discrete element models.

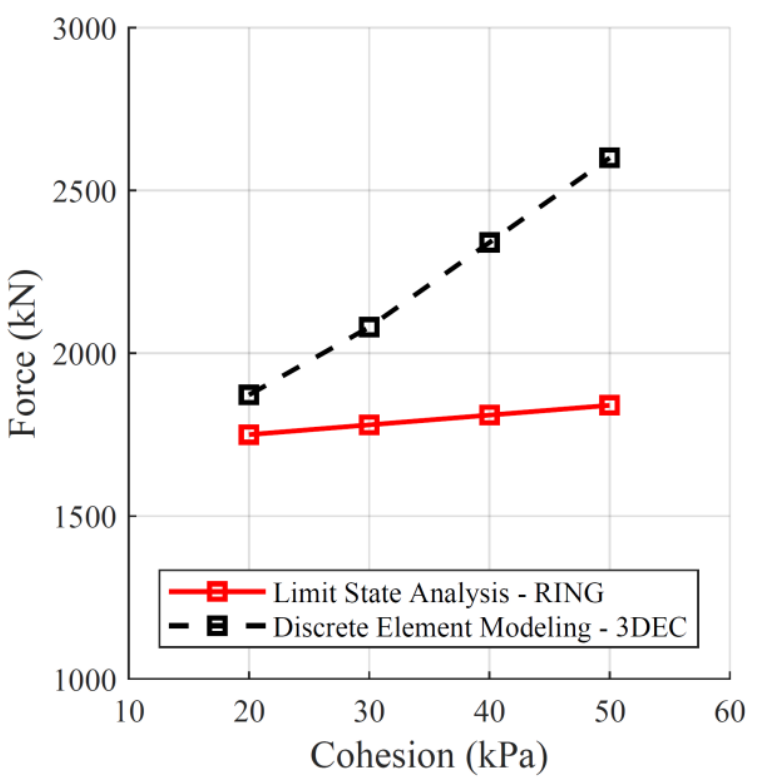

b) Influence of cohesion on the limit and discrete element models.

Figure 8. Influence of backfill properties (friction and cohesion) on the 2D limit state analysis and 3D discrete element models, for semi-shallow arch bridge with short span.

\subsection{Parametric Study on the Coulomb Joint Parameters between Masonry and Soil}

The presented advanced numerical modeling strategy has a clear advantage in terms of modeling of the cracks and large displacement with respect to other numerical methods. However, contact constitutive laws, assigned by the modeler, should be selected carefully since, they have a physical meaning and influence on the structure. The common contact law for discrete element modeling of masonry structures is the Coulomb joint model with tension cut-off. However, there is not much knowledge on the effect of contact parameters for the interaction between backfill material and masonry. Here, cohesion strength and friction angle are varied and load carrying capacity of the bridges are recorded.

It is observed that the friction angle has a pronounced influence on the overall capacity of masonry arch bridge, although, this did not change the failure mechanism due 
This is a pre-print version of an article published in Engineering Structures. Pulatsu, B., Erdogmus, E., \& Lourenço, P. B. (2019). Comparison of in-plane and out-of-plane failure modes of masonry arch bridges using discontinuum analysis. Engineering Structures, 178, 24-36.

to stiff form of the arch barrel (Table 4-2). On the other hand, cohesion strength demonstrated less influence, even no influence for shallow arch bridges, on the load carrying capacity compared to the friction angle.

Table 4-2 Contribution of the contact parameter, friction angle, between masonry and soil on the capacity of the masonry arch bridge (Cohesion is taken as zero).

\begin{tabular}{ccc} 
Bridge & Contact Friction Angle between M-S & Load Carrying Capacity $(\mathrm{kN})$ \\
\hline \multirow{3}{*}{ SSS } & 20 & 840 \\
& 30 & 960 \\
& 40 & 1000 \\
\hline \multirow{3}{*}{ SMS } & 20 & 960 \\
& 30 & 1175 \\
& 40 & 1248 \\
\hline \multirow{2}{*}{ DSS } & 20 & 865 \\
& 30 & 960 \\
& 40 & 1020 \\
\multirow{2}{*}{ DMS } & 20 & 1440 \\
& 30 & 1920 \\
& 40 & 2016 \\
\hline
\end{tabular}

\section{Discussion on Spandrel Wall Behavior}

The spandrel wall failures may be critical for the use of masonry arch bridges and for life safety. The spandrel wall failures may be observed in different forms depending on the interaction between masonry and soil backfill as well as the geometrical properties of the structure. The out-of-plumbness, sliding and bulging movements of the spandrel walls over the extrados of the arch barrel are the several structural defects observed during the survey, as shown in Figure 9. Furthermore, the excessive vegetation due to absence of mortar in the stone joints and longitudinal cracks in the arch barrel are the other most common problems $[40,41,42]$. 


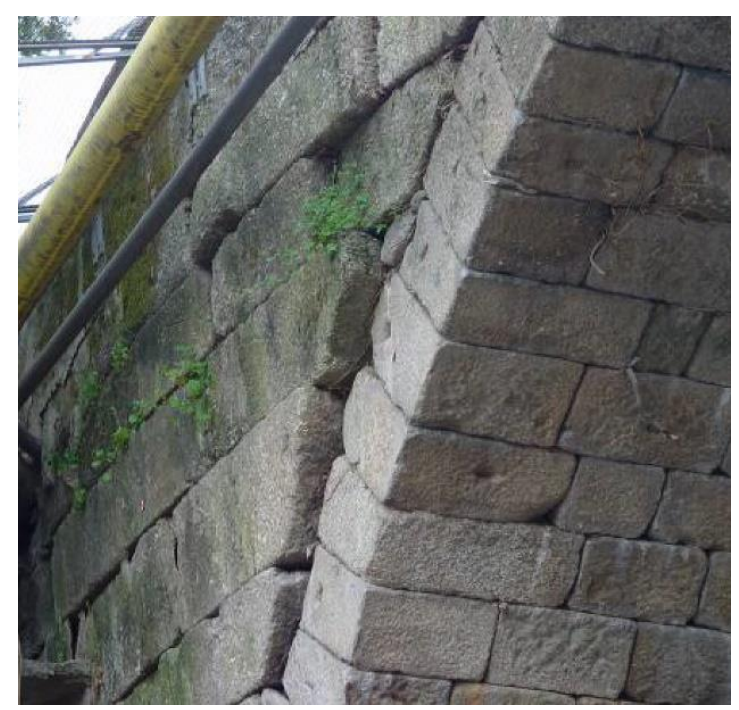

a) Sliding of the spandrel wall over the arch barrel.

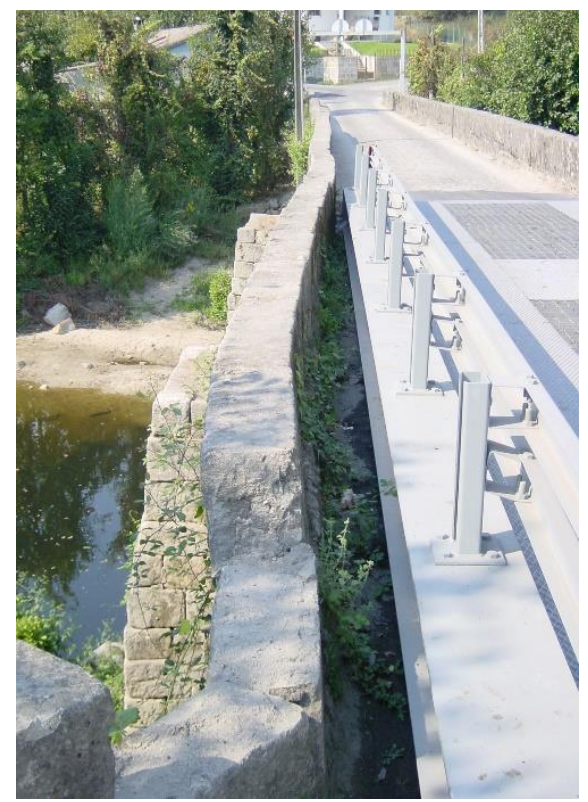

b) Out-of-plane deflection of the spandrel wall.

Figure 9. Spandrel wall deflections (Pont Donim, Portugal)

Spandrel wall analysis requires 3D models and complex numerical predictions that has been mostly done by macro-modeling approach, where the mechanical properties of masonry are smeared as a homogenous medium, to take the advantage of lower computational cost. Simplified processes have also been proposed to account for transverse actions in masonry arch bridges [42].

In this study, a more detailed approach is presented by considering a discontinuous displacement field in all masonry components of the arch bridge to provide realistic collapse mechanisms and more accurate load carrying capacity estimations. Both loading scenarios that are discussed previously are applied and the results show a remarkable out of plane displacement at the spandrel wall, which can yield up to $65 \%$ reduction on the load carrying capacity under the vehicle type of loading. The monitoring locations, on the spandrel wall and arch barrel, where the in-plane and out-of-plane displacements are recorded respectively, are shown for deep arch with medium span model in Figure 10. 
Obtained spandrel wall failures for deep and semi-shallow medium span arches are shown in Figure 11 and Figure 12, respectively. It is particularly noteworthy that the collapse mechanism of spandrel wall includes the combination of sliding of spandrel walls on the arch barrel and inevitable overturning failure. The damage progression in the numerical model started with the lateral sliding of the spandrel wall from the arch ring. Then, visible cracks appeared on the spandrel wall, which ended up with an overturning mechanism. The observed failure mechanism in the numerical model is aligned with a similar experimental test, presented by Boothby et al. [43]. Additionally, longitudinal cracks were observed at the intrados of the arch barrel due to soil pressure, close to the region where live loads were applied (Figure 13). Similar collapse mechanisms were observed for the other three representative masonry arch bridges as well. The presented numerical simulations highlight the importance of 3D behavior and necessity of detailed modeling to understand the structural behavior of the masonry arch bridges.

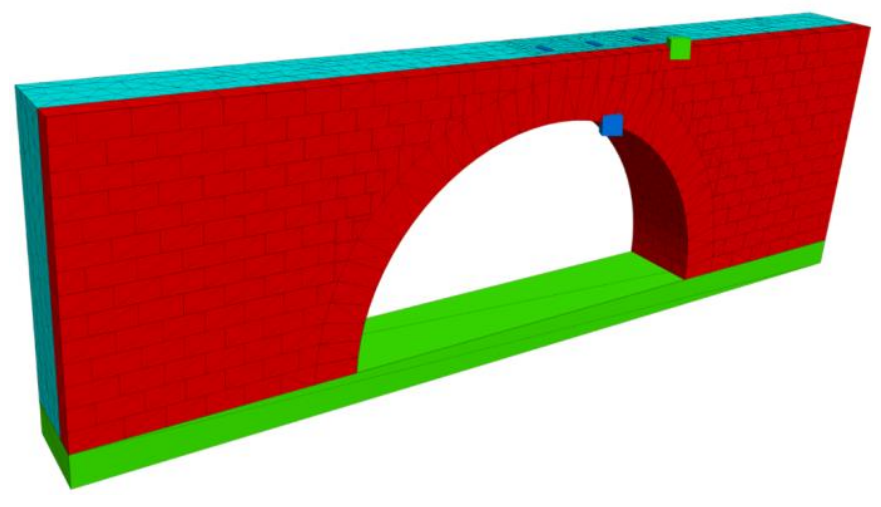

Figure 10. Monitoring points on arch barrel (R1) and spandrel wall (R2). 
This is a pre-print version of an article published in Engineering Structures. Pulatsu, B., Erdogmus, E., \& Lourenço, P. B. (2019). Comparison of in-plane and out-of-plane failure modes of masonry arch bridges using discontinuum analysis. Engineering Structures, 178, 24-36.

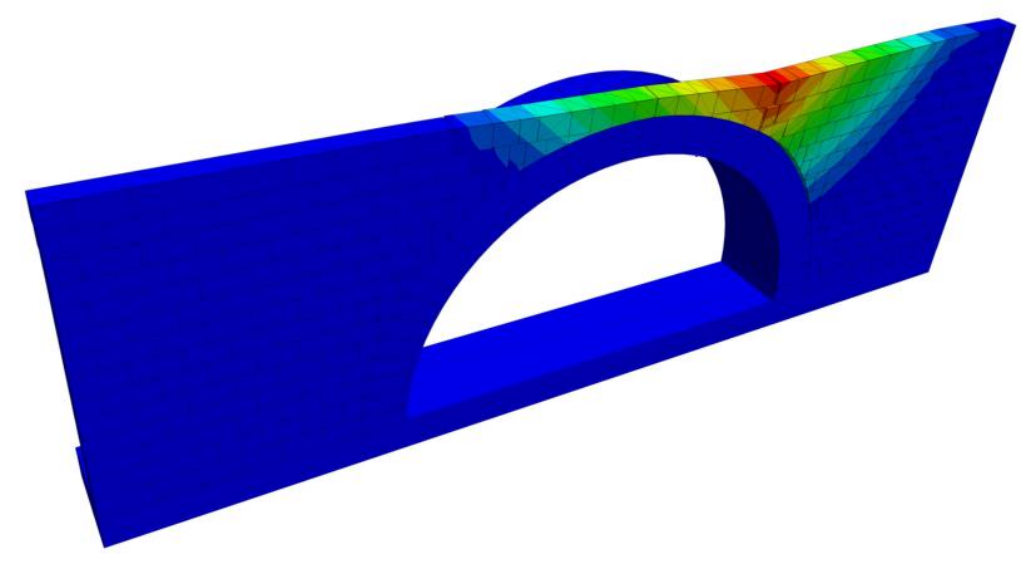

Figure 11. Spandrel wall failure of DMS (Colors display displacement magnitude)

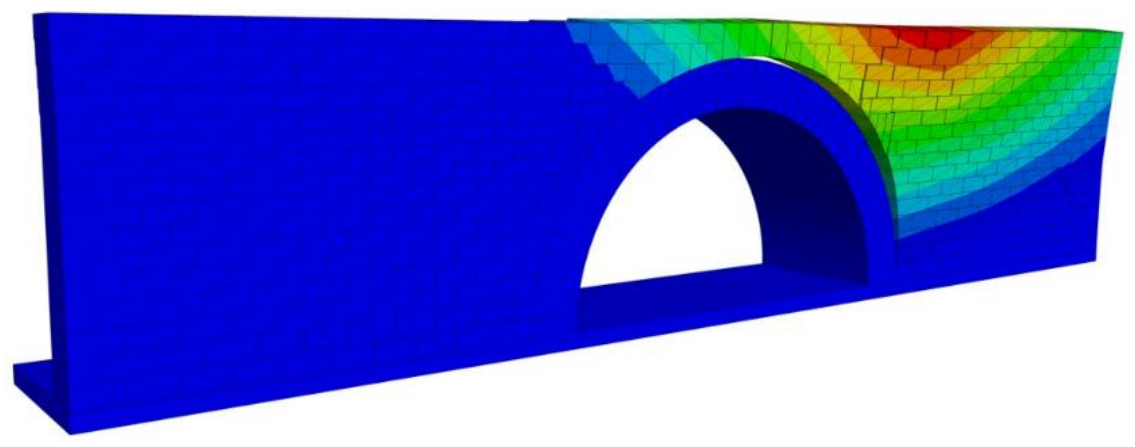

Figure 12. Spandrel wall failure of DSS (Colors display displacement magnitude). 


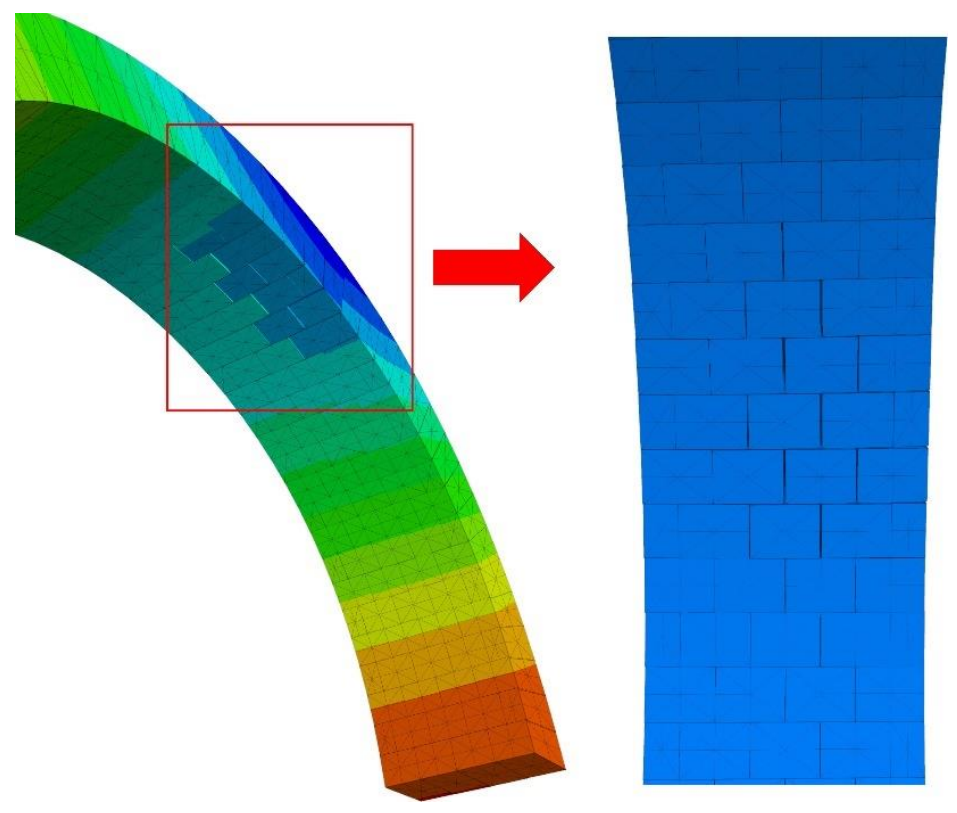

Figure 13. Out of plane displacement and the longitudinal cracks at the intrados of the arch barrel (Colors display displacement magnitude).

There is no significant effect of the contact parameters between masonry components of the examined arch bridges observed on the out-of-plane failure mode. However, in prepared case studies the spandrel wall height and the soil backfill depth is relatively low (0.4 $\mathrm{m}$ at the crown), which makes more likely to obtain sliding phenomena, rather than pure overturning. The capacity curves are given for all of the representative masonry bridges in Figure 14 under vehicle and knife edge loading. Results of the analysis indicates that the vehicle types of loading lead to a more critical force distribution on the spandrel wall than the knife edge loading and is more likely to cause a spandrel wall failure. It was noticed that loading plates, replicating the tire pressures, have a higher tendency to create transversal deflections of soil backfill (sliding) once the applied shear stress is higher than the shear strength of the backfill material. The arch barrel did not involve in the failure mechanism when bridges were subjected to vehicle loading. The out-of-plane deflection and the corresponding displacement field may be seen in Figure 15. Furthermore, the recorded spandrel wall deformations in the transversal direction are given in Figure 16. 
This is a pre-print version of an article published in Engineering Structures. Pulatsu, B., Erdogmus, E., \& Lourenço, P. B. (2019). Comparison of in-plane and out-of-plane failure modes of masonry arch bridges using discontinuum analysis. Engineering Structures, 178, 24-36.

On the other hand, knife edge loading yielded plastic hinging mechanism on the arch barrel and tensile cracks on the spandrel wall. Furthermore, there were limited spandrel wall deflections in the out-of-plane direction measured and the governing failure mode was a hinging mechanism of the arch barrel as shown in Figure 17. Therefore, the application of different type of pressures on the soil backfill will make critical response changes in masonry arch bridges. The results exhibit the lower and upper bounds for a masonry arch bridge, in which latter represents the collapse of arch barrel and former indicates the spandrel wall failure.

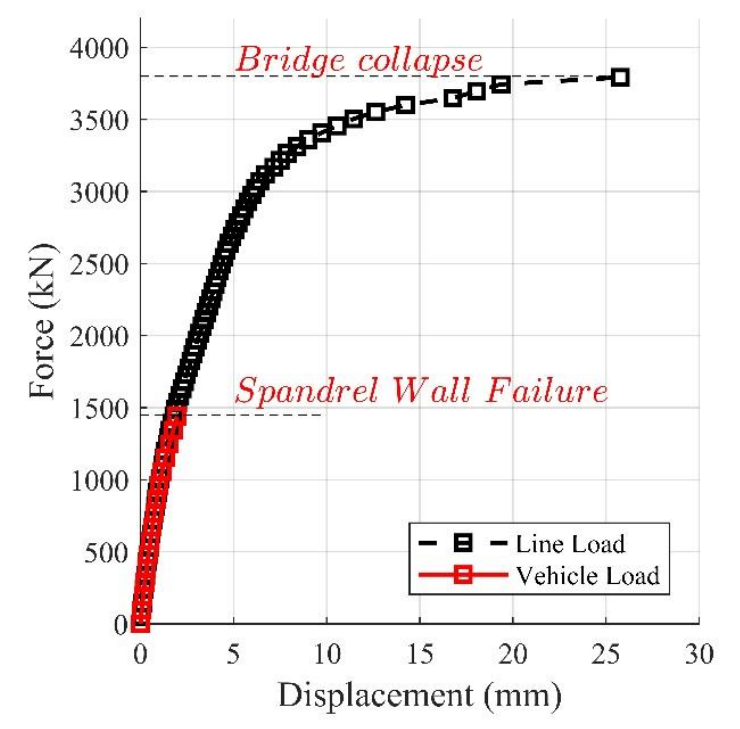

a) Force vs Displacement curves of Deep Arch with Medium Span Bridge (Deflection Recorded at the Arch Barrel, R1)

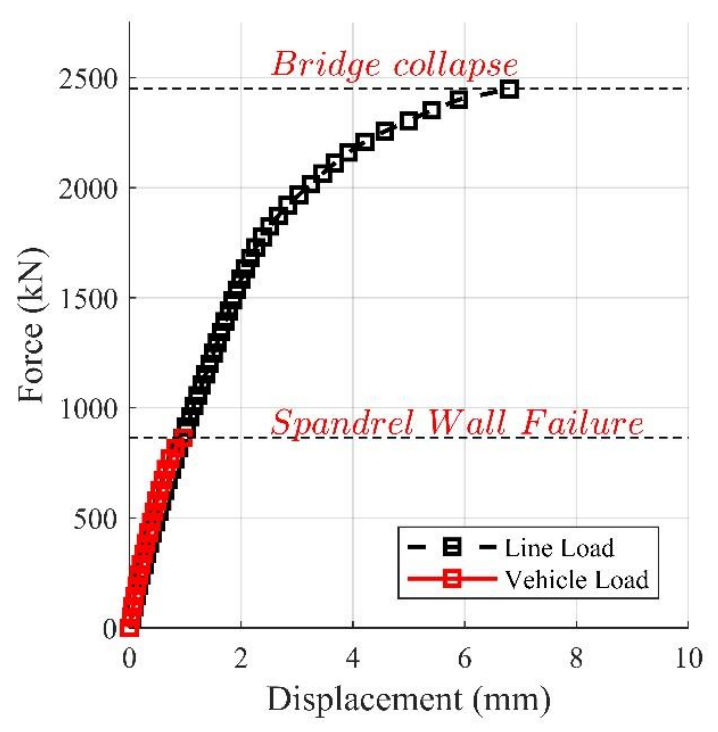

b) Force vs Displacement curves of Deep Arch with Sort Span Bridge (Deflection Recorded at the Arch Barrel, R1) 
This is a pre-print version of an article published in Engineering Structures. Pulatsu, B., Erdogmus, E., \& Lourenço, P. B. (2019). Comparison of in-plane and out-of-plane failure modes of masonry arch bridges using discontinuum analysis. Engineering Structures, 178, 24-36.

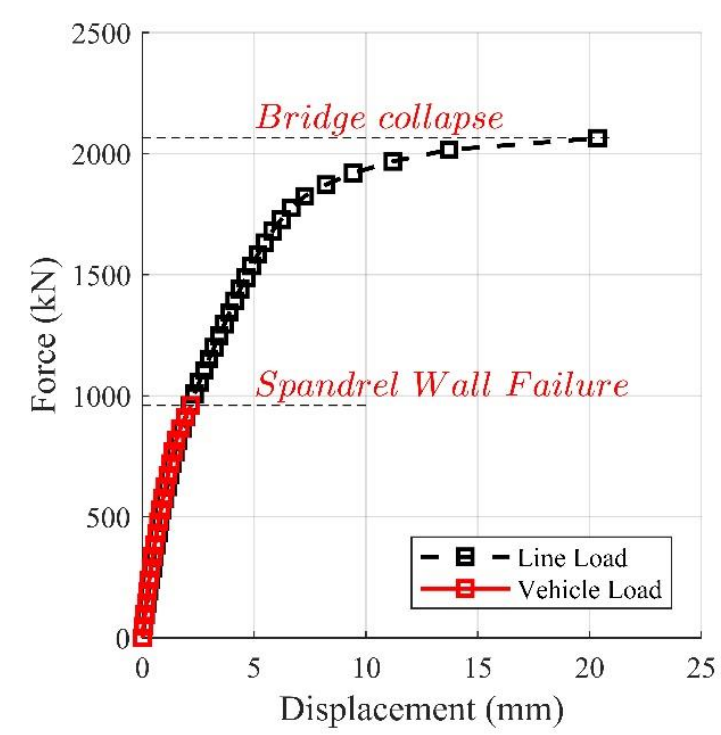

c) Force vs Displacement curves of SemiShallow Arch with Medium Span Bridge (Deflection Recorded at the Arch Barrel, $\mathrm{R} 1)$

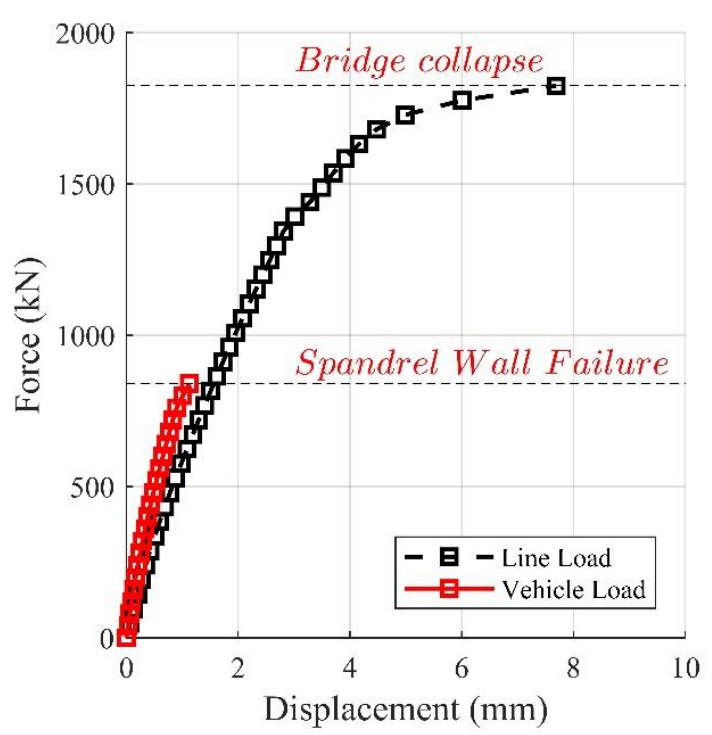

d) Force vs Displacement curves of SemiShallow Arch with Short Span Bridge (Deflection Recorded at the Arch Barrel, $\mathrm{R} 1)$

Figure 14. Capacity curves of four representative bridge model under two types of loading. 
This is a pre-print version of an article published in Engineering Structures. Pulatsu, B., Erdogmus, E., \& Lourenço, P. B. (2019). Comparison of in-plane and out-of-plane failure modes of masonry arch bridges using discontinuum analysis. Engineering Structures, 178, 24-36.

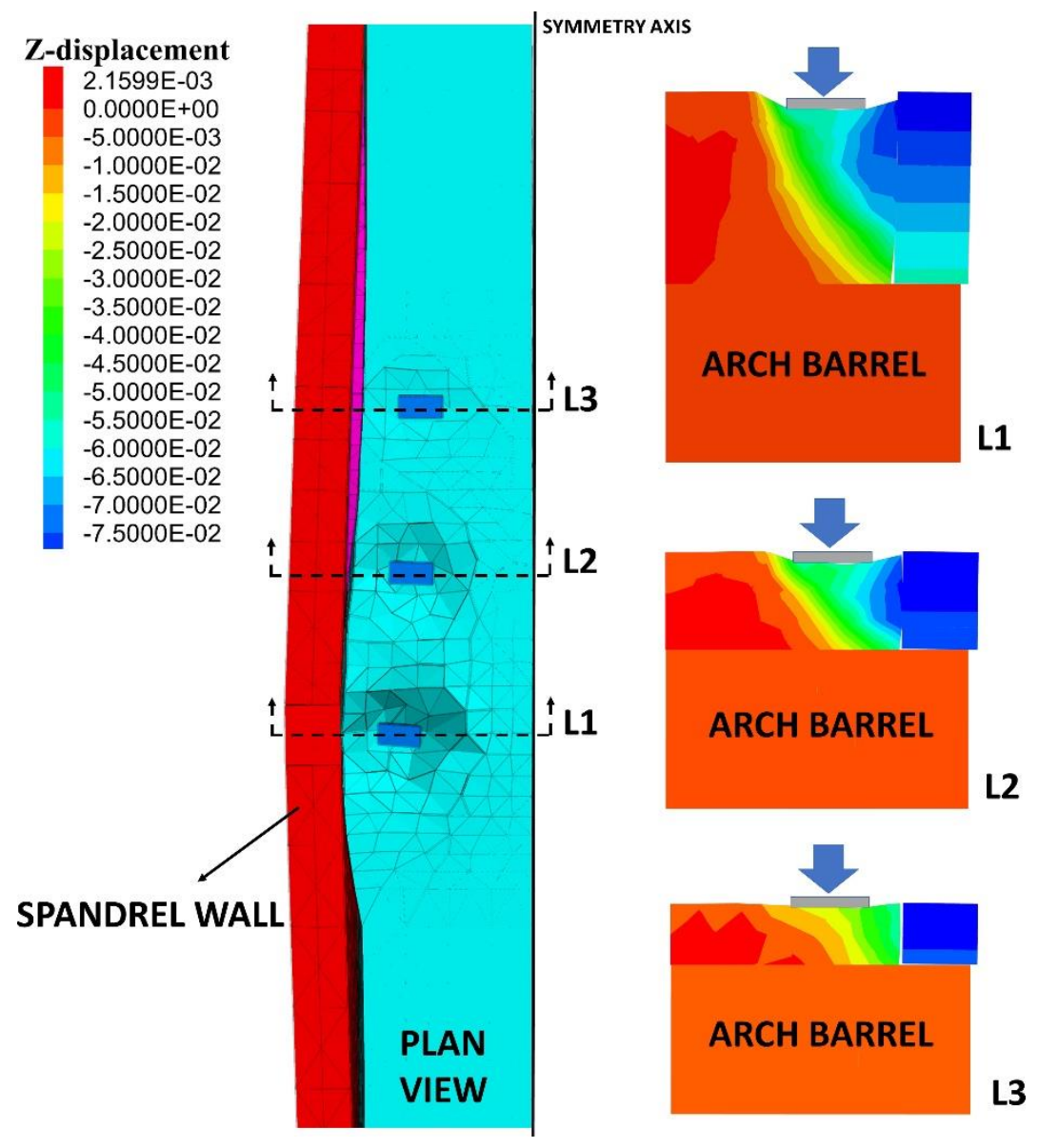

Figure 15. Deflection of spandrel wall (Plan view) and the out of plane displacement field at each cross section of loading plates under vehicle loading. 
This is a pre-print version of an article published in Engineering Structures. Pulatsu, B., Erdogmus, E., \& Lourenço, P. B. (2019). Comparison of in-plane and out-of-plane failure modes of masonry arch bridges using discontinuum analysis. Engineering Structures, 178, 24-36.

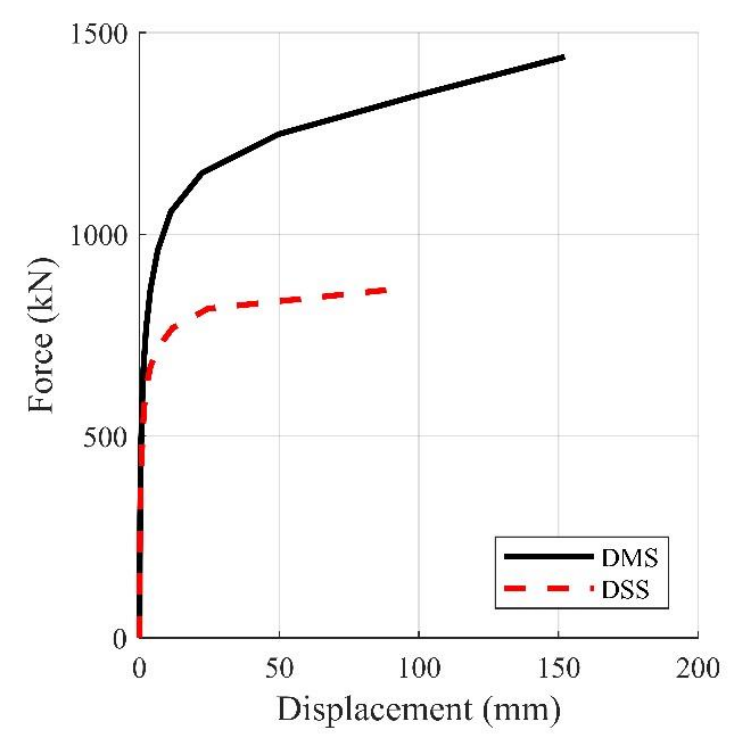

a) Deep Arch with Medium and Short Span Bridges under Vehicle Loading (Deflection Recorded at the Spandrel Wall, R2)

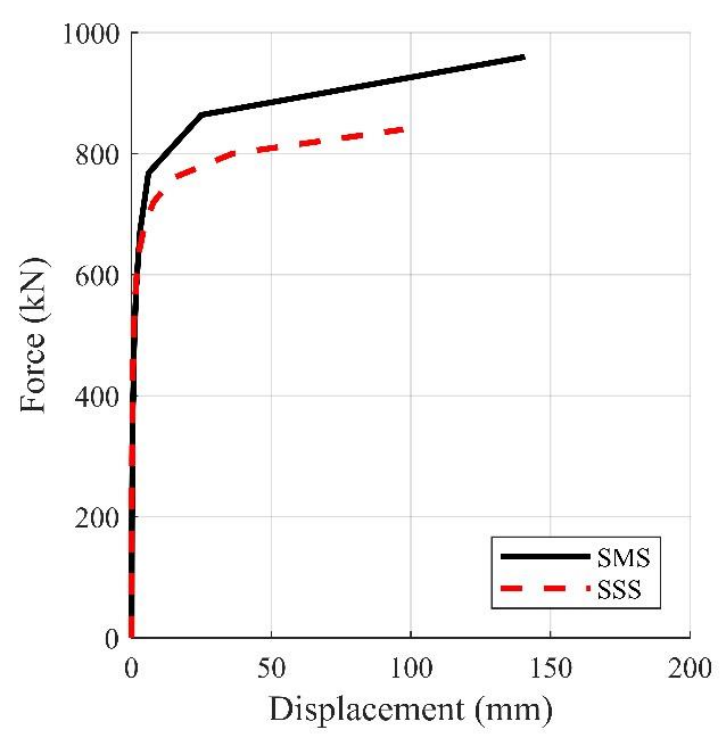

b) Semi-Shallow Arch with Medium and Short Span Bridges under Vehicle Loading (Deflection Recorded at the Spandrel Wall, R2)

Figure 16. Out of plane displacement of medium and short span masonry arch bridge spandrel walls under vehicle loading.

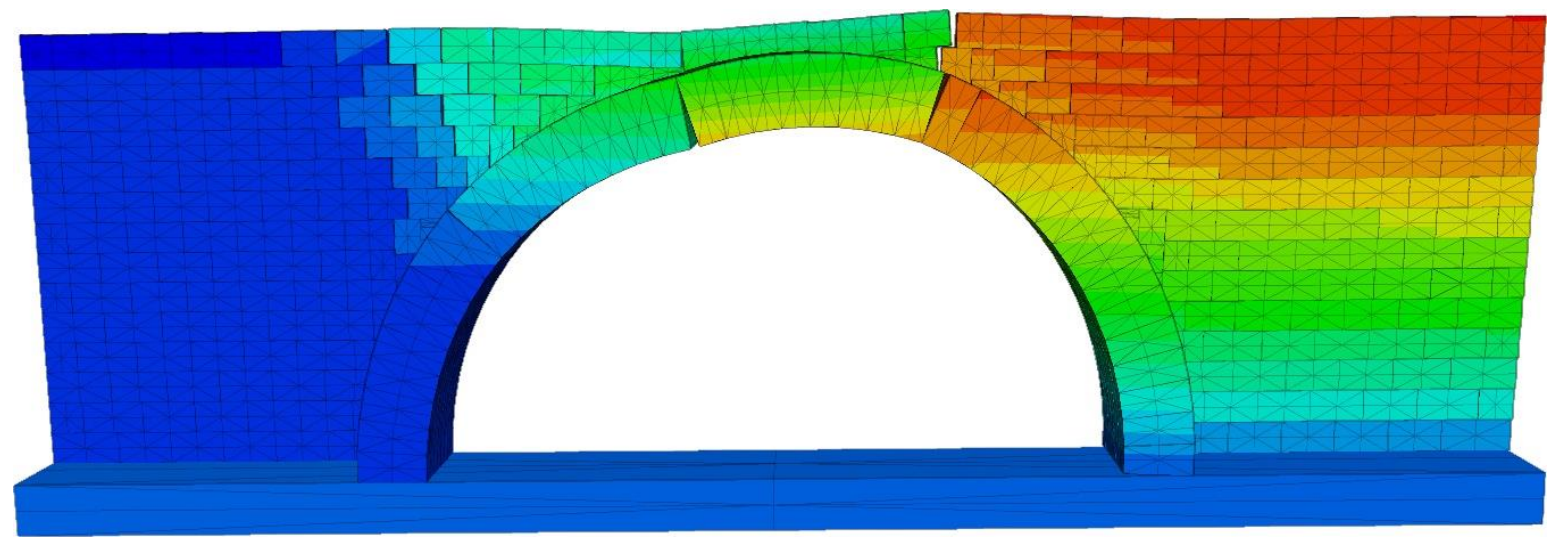

Figure 17. In-plane collapse mechanism of DMS under knife-edge loading (Colors display displacement magnitude). 


\section{Conclusions}

This research provides a better understanding of the complex behavior of masonry arch bridges by considering the influence of the backfill properties, contact between backfill and masonry, type of vehicle load assumed and spandrel wall vulnerability. First, the discontinuous and nonlinear characteristics of masonry bridges are simulated via a mixed discrete-continuum modeling strategy by conducting parametric analysis with a simplified approach, namely computational limit state analysis. A good agreement is found between the limit state analysis and the detailed numerical model for a shallow arch bridge with short span. In-plane collapse mode is obtained where masonry arch barrel failed due to plastic hinging mechanism. However, it is concluded that to understand the full structural response of masonry arch bridges, three-dimensional models are needed, which has the capability of simulating the transversal deflections, of the spandrel walls. From the advanced simulations, the following conclusions were derived:

(a) The cohesion of the backfill has a considerable influence on the capacity of masonry arch bridges, while friction of the backfill seems to have a lesser effect in comparison;

(b) The frictional resistance plays a key role at the interface between masonry and infill material, while the cohesion plays a less relevant role;

(c) The type of loading has a drastic impact on the capacity of masonry arch bridges. Under vehicle (point) loading, failure mode is governed by the spandrel wall failure. It is therefore noted that multiple concentrated loads provide different effect than a line load.

In addition, this research indicated the potential of mix discrete-continuum approach through the application on masonry arch bridges. In future studies, various structural problems of masonry arch bridges such as scour, and support settlement will be explored via this numerical modeling strategy. 
This is a pre-print version of an article published in Engineering Structures. Pulatsu, B., Erdogmus, E., \& Lourenço, P. B. (2019). Comparison of in-plane and out-of-plane failure modes of masonry arch bridges using discontinuum analysis. Engineering Structures, 178, 24-36.

\section{Acknowledgments}

Authors would like to express their gratitude to Itasca Educational Partnership Program (IEP) for their kind support and providing 3DEC software. Furthermore, authors would like to acknowledge the valuable help of Dr. Jim Hazzard, Itasca Consulting Group, for his technical support.

\section{References}

[1] Fanning PJ, Boothby TE, Roberts BJ. Longitudinal and transverse effects in masonry arch assessment. Constr Build Mater 2001;15:51-60. doi:10.1016/S09500618(00)00069-6.

[2] Tomor AK, McAulay S. Overview of design guidelines for masonry arch bridges. Struct Anal Hist Constr Anamn Diagnosis, Ther Control - Proc 10th Int Conf Struct Anal Hist Constr SAHC 2016 2016:598-603.

[3] Citto C, Woodham DB. Evaluating Existing and Historic Stone Arch Bridges. STRUCTURE 2015:14-8.

[4] Orbán Z. UIC Project on assessment, inspection and maintenance of masonry arch railway bridges. 5th Int Conf Arch Bridg 2007:3-12.

[5] Zucchini A, Lourenço PB. A micro-mechanical homogenisation model for masonry: Application to shear walls. Int J Solids Struct 2009;46:871-86. doi:10.1016/j.jijsolstr.2008.09.034.

[6] Boothby TE. Analysis of masonry arches and vaults. Prog Struct Eng Mater 2001;3:246-56. doi:10.1002/pse.84.

[7] Brencich A, De Francesco U. Assessment of Multispan Masonry Arch Bridges. I: Simplified Approach. J Bridg Eng 2004;9:582-90. doi:10.1061/(ASCE)10840702(2004)9:6(582).

[8] Brencich A, De Francesco U. Assessment of Multispan Masonry Arch Bridges II: Examples and Applications. J Bridg Eng 2004;9:591-8. doi:10.1061/(ASCE)10840702(2004)9:6(591).

[9] De Santis S, de Felice G. A fibre beam-based approach for the evaluation of the seismic capacity of masonry arches. Earthq Eng Struct Dyn 2014;43:1661-81. doi:10.1002/eqe.2416.

[10] Conde B, Drosopoulos GA, Stavroulakis GE, Riveiro B, Stavroulaki ME. Inverse analysis of masonry arch bridges for damaged condition investigation: Application on Kakodiki bridge. Eng Struct 2016;127:388-401. doi:10.1016/j.engstruct.2016.08.060.

[11] Betti M, Drosopoulos GA, Stavroulakis GE. Two non-linear finite element models developed for the assessment of failure of masonry arches. Comptes Rendus - Mec 2008;336:42-53. doi:10.1016/j.crme.2007.10.014. 
This is a pre-print version of an article published in Engineering Structures. Pulatsu, B., Erdogmus, E., \& Lourenço, P. B. (2019). Comparison of in-plane and out-of-plane failure modes of masonry arch bridges using discontinuum analysis. Engineering Structures, 178, 24-36.

[12] Zhang Y, Macorini M, Izzudin BA. Mesoscale Partitioned Analysis of Brick-Masonry Arches. Eng Struct 2016;to appear:142-66. doi:10.1016/j.engstruct.2016.05.046.

[13] Fanning PJ, Boothby TE. Three-dimensional modelling and full-scale testing of stone arch bridges. Comput Struct 2001;79:2645-62. doi:10.1016/S00457949(01)00109-2.

[14] Conde B, Ramos LF, Oliveira D V., Riveiro B, Solla M. Structural assessment of masonry arch bridges by combination of non-destructive testing techniques and three-dimensional numerical modelling: Application to Vilanova bridge. Eng Struct 2017;148:621-38. doi:10.1016/j.engstruct.2017.07.011.

[15] Senthivel R, Lourenço PB. Finite element modelling of deformation characteristics of historical stone masonry shear walls. Eng Struct 2009;31:1930-43. doi:10.1016/j.engstruct.2009.02.046.

[16] Lourenço PB, Rots JG. Multisurface interface model for analysis of masonry structures. J Eng Mech 1997;123:660-8.

[17] Lemos J V. Discrete Element Modeling of Masonry Structures. Int J Archit Herit 2007;1:190-213. doi:10.1080/15583050601176868.

[18] Lemos J. Assessment of the ultimate load of a masonry arch using discrete elements. Comput Methods Struct Mason 1995:294-302.

[19] Thavalingam A, Bicanic N, Robinson JI, Ponniah DA. Computational framework for discontinuous modelling of masonry arch bridges. Comput Struct 2001;79:182130. doi:10.1016/S0045-7949(01)00102-X.

[20] Bićanić N, Stirling C, Pearce CJ. Discontinuous modelling of masonry bridges. Comput Mech 2003;31:60-8. doi:10.1007/s00466-002-0393-0.

[21] Tóth AR, Orbán Z, Bagi K. Discrete element analysis of a stone masonry arch. Mech Res Commun 2009;36:469-80. doi:10.1016/j.mechrescom.2009.01.001.

[22] Sarhosis V, Oliveira DV, Lemos JV, Lourenco PB. The effect of skew angle on the mechanical behaviour of masonry arches. Mech Res Commun 2014;61:53-9. doi:10.1016/j.mechrescom.2014.07.008.

[23] Pulatsu B, Erdogmus E, Bretas EM. Parametric Study on Masonry Arches Using 2D Discrete Element Modeling. J Archit Eng 2018;24. doi:10.1061/(ASCE)AE.1943-5568.0000305.

[24] Roca P, Molins C. Experiments on arch bridges. In: Roca P, Molins C, editors. Arch Bridg. IV-Advances Assessment, Struct. Des. Constr., Barcelona: 2004, p. 365-74.

[25] Cundall PA, Hart RD. Numerical modelling of discontinua. Eng Comput 1992;9:101-13. doi:10.1108/eb023851.

[26] Cundall PA. Formulation of a three-dimensional distinct element model-Part I. A scheme to detect and represent contacts in a system composed of many polyhedral blocks. Int J Rock Mech Min Sci Geomech Abstr 1988;25:107-16.

[27] ITASCA. 3DEC Universial Discrete Element Code Theory and Background. Minneapolis: 2004. 
This is a pre-print version of an article published in Engineering Structures. Pulatsu, B., Erdogmus, E., \& Lourenço, P. B. (2019). Comparison of in-plane and out-of-plane failure modes of masonry arch bridges using discontinuum analysis. Engineering Structures, 178, 24-36.

[28] Simon J, Bagi K. Discrete element analysis of the minimum thickness of oval masonry domes. Int J Archit Herit 2014;3058. doi:10.1080/15583058.2014.996921.

[29] Pulatsu B, Bretas EM, Lourenco PB. Discrete element modeling of masonry structures: Validation and application. Earthquakes Struct 2016;11:563-82. doi:10.12989/eas.2016.11.4.563.

[30] Cocchetti G, Colasante G, Rizzi E. On the Analysis of Minimum Thickness in Circular Masonry Arches. Appl Mech Rev 2011;64:051002. doi:10.1115/1.4007417.

[31] Oliveira D V., Lourenço PB, Lemos C. Geometric issues and ultimate load capacity of masonry arch bridges from the northwest Iberian Peninsula. Eng Struct 2010;32:3955-65. doi:10.1016/j.engstruct.2010.09.006.

[32] Rota M, Pecker A, Bolognini D, Pinho R. A Methodology for Seismic Vulnerability of Masonry Arch Bridge Walls. J Earthq Eng 2005;9:331-53. doi:doi.org/10.1142/S1363246905002432.

[33] Riveiro B, Lourenço PB, Oliveira D V., González-Jorge H, Arias P. Automatic Morphologic Analysis of Quasi-Periodic Masonry Walls from LiDAR. Comput Civ Infrastruct Eng 2016;31:305-19. doi:10.1111/mice.12145.

[34] Livesley RK. Limit analysis of structures formed from rigid blocks. Int J Numer Methods Eng 1978;12:1853-71. doi:10.1002/nme.1620121207.

[35] LimitState. Ring manuel version 3.2.b. Sheffield: 2016.

[36] Callaway P, Gilbert M, Smith C. Influence of backfill on the capacity of masonry arch bridges. Proc ICE-Bridge Eng 2012;165:147-58. doi:10.1680/bren.11.00038.

[37] RSA. RSA Safety and Load Code for Buildings and Bridge Structures. Decreto Lei No. 235/83 [in Portuguese]. Lisboa, Portugal: 1983.

[38] Quezada JC, Vincens E, Mouterde R, Morel JC. 3D failure of a scale-down dry stone retaining wall: A DEM modelling. Eng Struct 2016;117:506-17. doi:10.1016/j.engstruct.2016.03.020.

[39] Milani G, Lourenço PB. 3D non-linear behavior of masonry arch bridges. Comput Struct 2012;110-111:133-50. doi:10.1016/j.compstruc.2012.07.008.

[40] Conde B, Díaz-Vilariño L, Lagüela S, Arias P. Structural analysis of Monforte de Lemos masonry arch bridge considering the influence of the geometry of the arches and fill material on the collapse load estimation. Constr Build Mater 2016;120:63042. doi:10.1016/j.conbuildmat.2016.05.107.

[41] Oliveira D V, Louren. Repair of stone masonry arch bridges. Arch Bridg - ARCH'04 2004:1-8.

[42] Erdogmus E, Boothby T. Strength of Spandrel Walls in Masonry Arch Bridges. Transp Res Rec J Transp Res Board 2004;1892:47-55. doi:10.3141/1892-06.

[43] Boothby TE, Yurianto Y, Erdogmus E. Experimental Replication of Masonry Arch Bridge Spandrel Wall Collapse. Mason Soc J 2005;23. 